\title{
Article
}

\section{Therapeutic Exercise Platform for Type-2 Diabetic Mellitus}

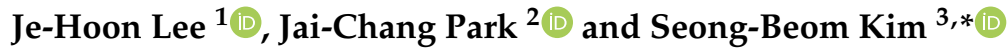 \\ 1 Division of Electronics, Information and Communication Engineering, Kangwon National University, \\ Samcheok-si 25913, Korea; jehoon.lee@kangwon.ac.kr \\ 2 Robot SW R\&D Team, Angel Robotics Co., Seoul 04798, Korea; jaichang@angel-robotics.com \\ 3 Department of Mechanical Design Engineering, Kangwon National University, Samcheok-si 25913, Korea \\ * Correspondence: sbkim81@kangwon.ac.kr; Tel.: +82-33-570-6396
}

check for

updates

Citation: Lee, J.-H.; Park, J.-C.; Kim, S.-B. Therapeutic Exercise Platform for Type-2 Diabetic Mellitus. Electronics 2021, 10, 1820. https:// doi.org/10.3390/electronics10151820

Academic Editor: J.-C. Chiao

Received: 20 June 2021

Accepted: 27 July 2021

Published: 29 July 2021

Publisher's Note: MDPI stays neutral with regard to jurisdictional claims in published maps and institutional affiliations.

Copyright: (c) 2021 by the authors. Licensee MDPI, Basel, Switzerland. This article is an open access article distributed under the terms and conditions of the Creative Commons Attribution (CC BY) license (https:// creativecommons.org/licenses/by/ $4.0 /)$.

\begin{abstract}
Exercise enables continuous glycemic control for diabetic patients, and it is effective in preventing diabetic complications and maintaining emotional stability. However, it is difficult for diabetic patients to know the appropriate intensity and duration of exercise. Excessive exercise causes sudden hypoglycemia, and patients avoid therapeutic exercise or perform it conservatively owing to the repeated hypoglycemia symptoms. In this paper, we propose a new therapeutic exercise platform that supports type 2 diabetes patients to exercise regularly according to the exercise prescription received from the hospital. The proposed platform includes the following three significant contributions. First, we develop a hardware platform that automatically tracks and records all aerobic exercise performed by a patient indoors or outdoors using a wearable band and aerobic exercise equipment. Second, we devise a patient-specific exercise stress test to know whether the patient is exercising according to his or her usual exercise regimen. Finally, we develop a mobile application that informs patients in real-time whether they are exercising appropriately for their exercise regimen each time they exercise. For platform evaluation and future improvement, we received satisfaction ratings and functional improvements through a questionnaire survey on 10 type 2 diabetes patients and 10 persons without a diabetes diagnosis who had used the proposed platform for more than 3 months. Most users were (1) satisfied with automatic exercise recording, and (2) exercise time increased. Diabetics reported that their fasting blood glucose was dropped, and they were more motivated to exercise. These results prove that exercise must be combined with medication for blood glucose management in chronic diabetic patients. The proposed platform can be helpful for patients to continue their daily exercise according to their exercise prescription.
\end{abstract}

Keywords: therapeutic exercise; diabetes; self-care management; self-monitoring blood glucose; exercise stress test; mobile health

\section{Introduction}

Type 1 diabetes is caused by a lack of insulin, which controls blood glucose levels in the body due to inherited genetic traits, lifestyle, and aging. Type 2 diabetes is caused by a combination of resistance to insulin action and an inadequate compensatory insulin secretory response. The number of type 2 diabetic patients is increasing rapidly because of the increasing elderly population and lifestyle changes. Complications are more dangerous than diabetes itself, such as kidney disease, retinopathy, and neurological disorders. Diabetic patients commonly experience emotional stress when blood glucose management is neglected, such as anxiety, depression, and regret [1,2]. Mental stress and physical pain make the patient's quality of life worse when diabetes develops into a chronic disease. It is essential to develop a convenient and accurate blood glucose management program that helps diabetic patients to manage their blood glucose levels by themselves [3,4].

The purpose of diabetes treatment is to keep blood glucose levels within the normal range, that is, diabetes self-management. The key is balancing between lifestyle habits that affect blood glucose. The patient should exercise regularly, control diet to maintain 
adequate calories, and take diabetes medications. Blood glucose monitoring can help the patient understand the link between blood glucose, food, exercise, and medication. Furthermore, the patients should try to improve their poor lifestyle continuously.

Most diabetic patients take their diabetes medications on time. The patients who manage blood glucose well should record blood glucose readings before and after meals and the amount of food consumed. It will help balance food intake and blood glucose levels. In addition, exercise is another lifestyle that significantly affects blood glucose levels. However, it is hard to record precisely the exercise performed by the patient and to calculate the patient's appropriate amount of exercise. Although there have been many studies on the relationship between exercise and blood glucose levels for decades, the number of mobile applications that patients can use for blood glucose management are few or limited.

A therapeutic exercise significantly affects the patient's emotional stability, reducing the risk of cardiovascular disease which is related to complications. However, it is difficult for diabetic patients to know the appropriate intensity and duration of exercise due to fear of hypoglycemia caused by excessive exercise and different physical conditions for each individual. Therefore, most diabetic patients are less aware of the importance of therapeutic exercise [5]. Most previous studies about therapeutic exercise have attempted to analyze the effect of exercise on blood glucose levels [6-10]. They attempted to determine the appropriate intensity, duration, frequency of exercise for blood glucose management in diabetics. Several studies considered exercise therapy for diabetic patients, focusing mainly on aerobic exercise using a treadmill, ergometer, or stepper; or exercising without a device, such as walking or running.

A study using an ergometer showed a comparison of the reduction in blood glucose levels after exercise in healthy people and diabetics [11]. According to this study, both diabetic patients and persons without a diabetes diagnosis have lower blood glucose levels after exercise than pre-exercise blood glucose levels. However, after 60 minutes of exercise in persons without a diabetes diagnosis, blood glucose levels were not significantly different from before exercise. In contrast, after 60 minutes of exercise in diabetic patients, blood glucose levels were up to $80 \%$ before exercise. This fact indicates that exercise helps people with diabetes manage their blood glucose levels. A study on blood glucose response concerning exercise intensity for type 1 diabetes patients [12] showed that high-intensity exercise made patients more sensitive to exercise intensity immediately after exercise. It also presented that moderate-intensity and intermittent high-intensity exercise program reduces blood glucose levels more effectively than continuous moderate-intensity exercise. Resistance exercise using a band or similar device has also been effective, particularly for type 2 diabetic patients who have difficulty with aerobic exercise [13].

Although most patients agreed that they should perform regular exercise individually at home, research on developing a continuous healthcare system supporting this is insufficient. This paper proposes a therapeutic exercise platform to help diabetic patients exercise with appropriate exercise intensity when walking or running outdoors or using aerobic exercise equipment indoors. Patients diagnosed with diabetes receive an appropriate exercise prescription in the hospital. As time passes after receiving an exercise plan, the patient's condition changes. It is difficult for the patient to know the appropriate exercise intensity.

The proposed platform provides an exercise program to achieve prescribed exercise goals. It automatically obtains heart rate and exercise information during exercise session and supports a real-time monitoring mode to ensure that the patient is exercising correctly. In addition, a patient's health and fitness will change over time. The proposed platform also provides an exercise stress test mode to adjust the exercise intensity according to the current patient's condition. Figure 1 shows the exercise sequence of the proposed platform.

(1) The patient visits a hospital and receives an exercise prescription after an exercise stress test. It includes the type of exercise, specific workloads, duration, and frequency of exercise sessions for their blood glucose management. 
(2) The proposed platform sets the patient's exercise program according to the exercise prescription. Then, it provides the program during the exercise session.

(3) The patient exercises during the exercise session according to the exercise program. The proposed platform automatically obtains heart rate and exercise information and provides them in real-time to check their exercise.

(4) At the end of the exercise session, the proposed platform evaluates whether the patient exercised according to the exercise prescription. If the difference is significant, the platform fine-tunes the exercise program to maintain the original exercise prescription with additional exercise stress testing.

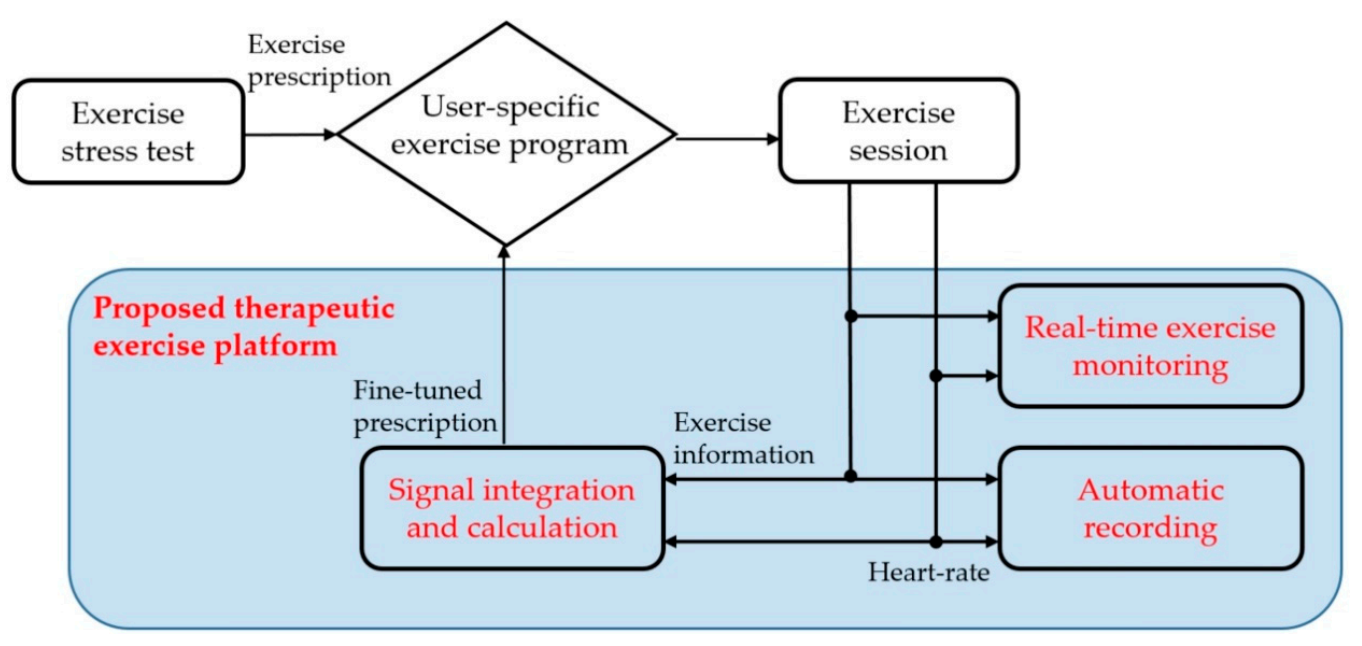

Figure 1. The proposed scheme of the proposed therapeutic exercise platform.

As a result, the proposed platform helps the patient exercise while maintaining the initial goal by adjusting the exercise program, even if the patient's condition or exercise sensitivity changes from when receiving an exercise prescription. This paper presents the hardware configuration, the exercise stress test for adjustment of the exercise program, and the user application of the proposed platform.

To develop this platform, we built a hardware system that monitors patient movement indoors and outdoors. The proposed platform analyzes the patient's exercise and adjusts the exercise intensity according to the result to provide the patient with an exercise session. A total of 10 diabetic patients and 10 persons without a diabetes diagnosis used the proposed platform for 3 months, and we analyzed the effectiveness of the proposed platform through their survey.

\section{Therapeutic Exercise and Blood Glucose Management}

Most self-managed programs for diabetes support balancing between physical exercise, insulin dosage or medication, blood glucose monitoring, and diet. Each patient has different physical strength, and the effect of exercise on blood glucose levels is also different. Therefore, most patients consider exercise as a supplemental glycemic control method. It is essential to study the effect of exercise on blood glucose levels to use exercise as an effective glycemic control method. In particular, it is crucial to develop a therapeutic exercise program that can recommend an exercise suitable for glycemic control to patients according to their preferred exercise pattern and individual exercise ability.

For decades, many kinds of research have been conducted to use therapeutic exercise for blood glucose management [14-22]. The aim of therapeutic exercise for diabetic patients is to increase insulin sensitivity. It is to mitigate or ideally to prevent diabetes complications. Insulin secretion decreases as the prevalence period of diabetes increases and fasting plasma glucose increases. Pan et al. presented that long-term regular exercise can improve lipid metabolism by reducing blood glucose levels and increasing insulin sensitivity to muscles, thereby improving various risk factors such as arteriosclerosis [14]. Oshida 
et al. recommended that light aerobic exercise improves the insulin signaling pathway. Additionally, they concluded that aerobic exercise, including resistance one, can improve insulin resistance and quality of life [15].

Table 1 summarizes recent findings regarding how long-term regular aerobic and resistance exercises help manage patients' blood glucose levels [16-19].

Table 1. Research comparisons according to exercise type, intensity, and frequency.

\begin{tabular}{|c|c|c|c|c|c|}
\hline Study & Sample & Intervention & $\begin{array}{c}\text { Measurement of } \\
\text { PA }\end{array}$ & $\begin{array}{l}\text { Physiological } \\
\text { Outcome }\end{array}$ & $\begin{array}{c}\text { Main } \\
\text { Findings/Conclusions }\end{array}$ \\
\hline $\begin{array}{l}\text { Adolfsson et al. } \\
\qquad \begin{array}{c}\text { (2012) } \\
\text { Sweden }\end{array}\end{array}$ & $\mathrm{N}=24$ & $\begin{array}{c}\text { Aerobic exercise of } \\
\text { different intensities } \\
\text { on } 3 \text { consecutive } \\
\text { days }\end{array}$ & Ergometer & $\begin{array}{c}\mathrm{VO}_{2 \max } \text { (maximal } \\
\text { oxygen } \\
\text { consumption) }\end{array}$ & $\begin{array}{l}\text { Physical capacity in } \\
\text { connection with physical } \\
\text { exercise of different } \\
\text { intensities did not differ } \\
\text { between adolescents } \\
\text { with diabetes. }\end{array}$ \\
\hline $\begin{array}{c}\text { Chiang et al. (2019) } \\
\text { Taiwan }\end{array}$ & $\mathrm{N}=20$ & $\begin{array}{l}\text { Aerobic exercise of } \\
\text { moderate } \\
\text { intensities on } 12 \\
\text { weeks }\end{array}$ & Treadmill & $\begin{array}{c}\mathrm{VO}_{2 \text { max }}, \mathrm{HgbA1c}, \\
\mathrm{BG} \text { level }\end{array}$ & $\begin{array}{c}\text { A } 12 \text { weeks } \\
\text { moderate-intensity } \\
\text { exercise training appears } \\
\text { safe for type } 2 \\
\text { diabetes patients. }\end{array}$ \\
\hline $\begin{array}{c}\text { Najafabadi et al. } \\
(2021) \\
\text { Iran }\end{array}$ & $\mathrm{N}=15$ & $\begin{array}{l}\text { Aerobic exercise of } \\
\text { moderate } \\
\text { intensities (three } \\
\text { sessions per week } \\
\text { for } 8 \text { weeks) }\end{array}$ & Walking & $\begin{array}{c}\text { BMI, HbA1c, } \\
\text { triglyceride, total } \\
\text { cholesterol, etc. }\end{array}$ & $\begin{array}{c}\text { AE (Aerobic exercise) } \\
\text { played an important role } \\
\text { in reducing HbA1c and } \\
\text { FBS (fasting blood } \\
\text { sugar) levels. }\end{array}$ \\
\hline $\begin{array}{l}\text { Moniotte et al. } \\
\text { (2017) } \\
\text { Belgium }\end{array}$ & $\mathrm{N}=24$ & $\begin{array}{l}\text { Two sessions of } 30 \\
\text { min of MVPA } \\
\text { (moderate to } \\
\text { vigorous physical } \\
\text { activity) }\end{array}$ & Treadmill & $\begin{array}{l}\text { BG level and } \\
\text { subcutaneous } \\
\text { glucose level }\end{array}$ & $\begin{array}{l}\text { Algorithmic adaptations } \\
\text { of insulin doses were } \\
\text { associated with better } \\
\text { outcomes in terms of } \\
\text { post-exercise } \\
\text { glucose control. }\end{array}$ \\
\hline
\end{tabular}

Diabetic patients should perform exercise stress tests and receive exercise prescriptions before starting exercise. Resistance exercise is a fundamental factor in exercise prescription constituted by exercise modality. In this paper, only aerobic exercise is focused as the main exercise factor. The three essential factors in exercise prescription are the intensity, duration, and frequency of exercise. These are all quantitative conditions, and highlighting one condition can increase the effectiveness of exercise. In particular, the most important thing is the exercise intensity [23].

Many studies have been conducted to determine the appropriate exercise intensity. In general, exercise intensity should be based on the anaerobic threshold measured by cardiopulmonary exercise testing (CPX) [24]. The test is both expensive and time-consuming, and most exercise facilities do not have a respiratory gas analyzer. Instead of CPX, it is possible to set the desired training intensity, expressed as a \% of maximum heart rate, $H R_{\max }$, with the Karvonen formula.

First, target heart rate (THR) is obtained from Karvonen's formula. The maximum heart rate is roughly estimated using Equation (1). The heart rate reserve (HRR) is obtained from subtracting resting heart rate, $H R_{\text {rest }}$, from $H R_{\max }$ as Equation (2). Target heart rate is calculated from predicted heart rate reserve, exercise intensity, and resting heart rate using Equation (3).

$$
\begin{gathered}
H R_{\max }=220-\text { age } \\
H R R=H R_{\text {max }}-H R_{\text {rest }} \\
T H R=[H R R \times \text { Exercise intensity }]+H R_{\text {rest }}
\end{gathered}
$$


where Exercise intensity is the constant number of exercise intensity and is the coefficient determined by a value ranging from 0.3 to 0.8 based on the desired exercise intensity.

$H R_{\max }$ describes the highest heart rate achieved by a subject exercising to exhaustion and is verified by a plateau of heart rate [25]. In the literature, $H R_{\max }$ refers to the peak heart rate at the termination of a graded maximal exercise test $[26,27]$. $H R_{\max }$ predicted by age alone may be practically convenient for the various groups. However, the magnitude and possible modifying effect are not fully established. Nes et al. presented that $H R_{\max }$ was linearly and inversely related to age explained by the formula; $211-(0.64 \times$ age $)$. Gellish et al. suggested $206.9-(0.67 \times$ age $)$, which has become the de-facto standard approach to determine maximum heart rate [28].

The simplest method is the Bogs scale method, which scores the feeling of difficulty during exercise: 13 = a little hard, $15=$ hard, etc. However, the myocardial burden, i.e., the burden on the heart depending on aerobic exercise type, cannot be the same for all patients, even if the exercise prescription sets the correct exercise intensity based on heart rate or maximum oxygen intake or user awareness.

Exercise intensity for diabetic patients is evaluated by the amount of oxygen intake or heart rate during an exercise stress test. The American Diabetes Association recommends that appropriate exercise intensity for diabetic patients is $50-70 \%$ of $H R_{\max }$, whereas the Japanese Medical Association recommends $40-60 \%$ of $H R_{\max }$ as optimal exercise intensity for type 2 diabetics [29]. Appropriate exercise intensity in Korean diabetic patients was determined after deriving patient $H R_{\max }$ or maximum oxygen intake through an exercise stress test [30]. Regression analysis using data from 581 diabetic patients determined by Equation (4).

$$
H R_{\text {max }}=172-0.625 \times \text { age }
$$

MET (metabolic equivalents) and RPE (ratings of perceived exertion) must be considered for each exercise device to include individual deviations. Exercise duration and frequency must also be considered, and exercise type and duration should be tailored to individual patient circumstances.

The exercise test is mainly performed on aerobic exercise equipment, such as ergometers and treadmills. Treadmills provide exercise load with walking slope and speed, while ergometers provide exercise load with pedal resistance and speed. Vital signs, such as blood pressure and heart rate, are measured during the exercise stress test. Blood glucose levels are also monitored before and after exercise to analyze the relationship between exercise load and blood glucose levels.

Several studies have shown that blood glucose levels decrease during high or mediumintensity exercise $[13,31-34]$. The intermittent high-intensity exercise produced more significant blood glucose level reduction than continuous moderate-intensity exercise. Blood glucose reduction due to exercise was proportional to exercise intensity and time. Thus, moderate and intermittent high-intensity exercise for 45 minutes or more is beneficial for blood glucose than high-intensity exercise for a short period.

In general, when diabetes is diagnosed, the appropriate amount of exercise considering the patient's physical strength is determined through CPX. However, if the patient repeats the same intensity for a long time, the patient's exercise ability should be improved. In practice, a method other than CPX is needed to predict AT (anaerobic threshold) and determine exercise intensity. The best way is to develop an exercise platform that allows patients to determine the exercise intensity using their aerobic exercise equipment easily. While the patient is exercising like always, the exercise information and the heart rate are obtained automatically. After the exercise intensity is determined using the obtained information, the result is notified to the patient. Because the patient can test easily, exercise tests can be performed whenever they feel that their exercise ability has changed. Consequently, it can help the patient quickly know the appropriate exercise intensity. 


\section{Proposed Therapeutic Exercise Platform}

This study proposes a system that supports diabetic patients to manage their blood glucose levels using the proposed therapeutic exercise platform, as shown in Figure 2. The proposed system has three main functions.

Proposed therapeutic exercise $\mathrm{H} / \mathrm{W}$ configuration

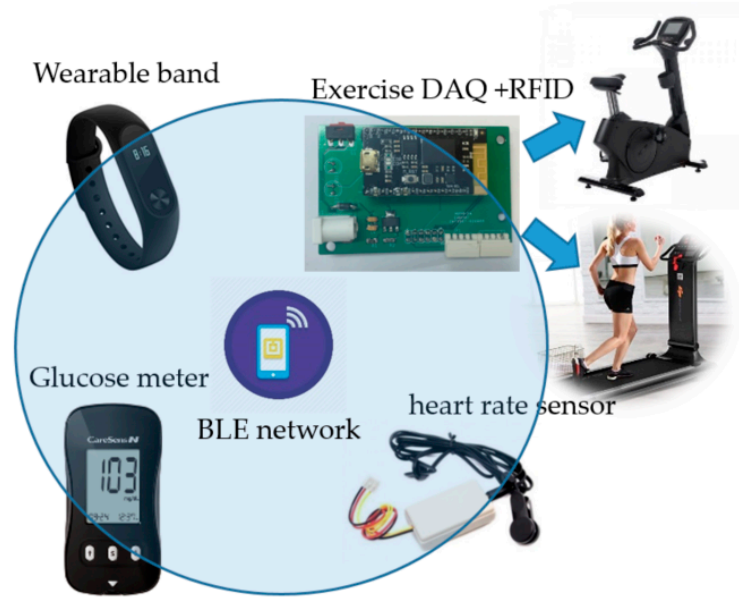

(a)

Proposed therapeutic exercise application

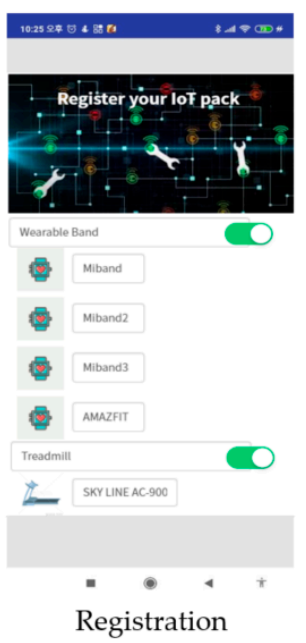

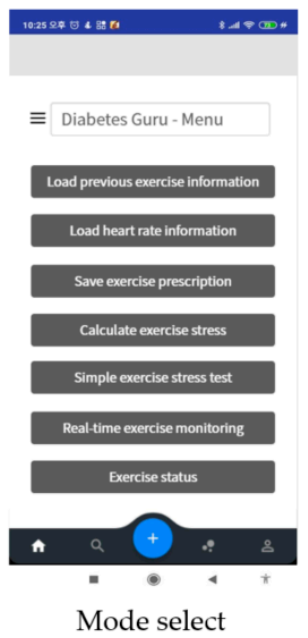

(b)

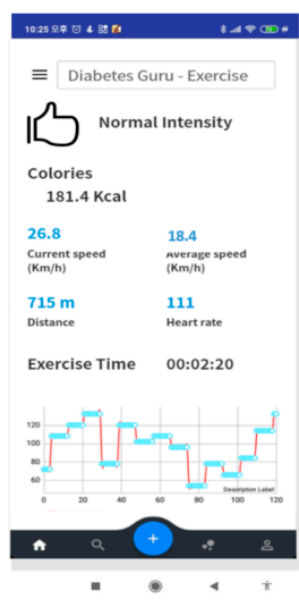

Real-time exercise monitoring

Figure 2. The proposed therapeutic exercise platform: (a) Hardware configuration; (b) Smartphone application of the proposed platform.

1. Automatically obtain exercise information whenever patients exercise outdoors or indoors.

2. Analyze the change of exercise ability due to the long-term exercise regime, i.e., a simple exercise stress test that allows the user to test their athletic ability.

3. Provide exercise coaching that informs the user whether they are exercising appropriately during a session.

The proposed exercise platform is composed mainly of hardware parts and smartphone applications. The hardware part serves to acquire exercise information and heart rate during exercise automatically. The smartphone application provides a simple exercise test and real-time exercise monitoring results using the hardware part's information. Each of them is described in detail below.

\subsection{Hardware Configuration of the Proposed Therapeutic Exercise Platform}

The proposed platform supports therapeutic exercise for blood glucose management based on aerobic options, i.e., jogging and walking outdoors and using a stationary bike or treadmill indoors. During the exercise session, the proposed platform automatically acquires exercise information such as exercise type, speed, and exercise time. It transfers the data to the proposed smartphone application in real-time.

The hardware configuration of the proposed platform includes five elements, as shown in Figure 2a. First, it uses a radio frequency identification (RFID) module to identify the user, which is embedded into the exercise equipment. Second, it uses a wearable band or ear-clip heart rate sensor to check heart rate during the exercise session. Third, the wearable band is responsible for monitoring outdoor exercise, including the heart rate of patients. Fourth, an exercise data acquisition (DAQ) device is added to the exercise devices to monitor the indoor aerobic exercise. Finally, glucometers are used to record the blood glucose readings. Figure 3 shows the data flow of the proposed therapeutic exercise platform. The collected data is calculated as necessary information, and then it is 
stored in the internal memory of each device. When the smartphone and each device are synchronized, this information is transmitted to the smartphone application.

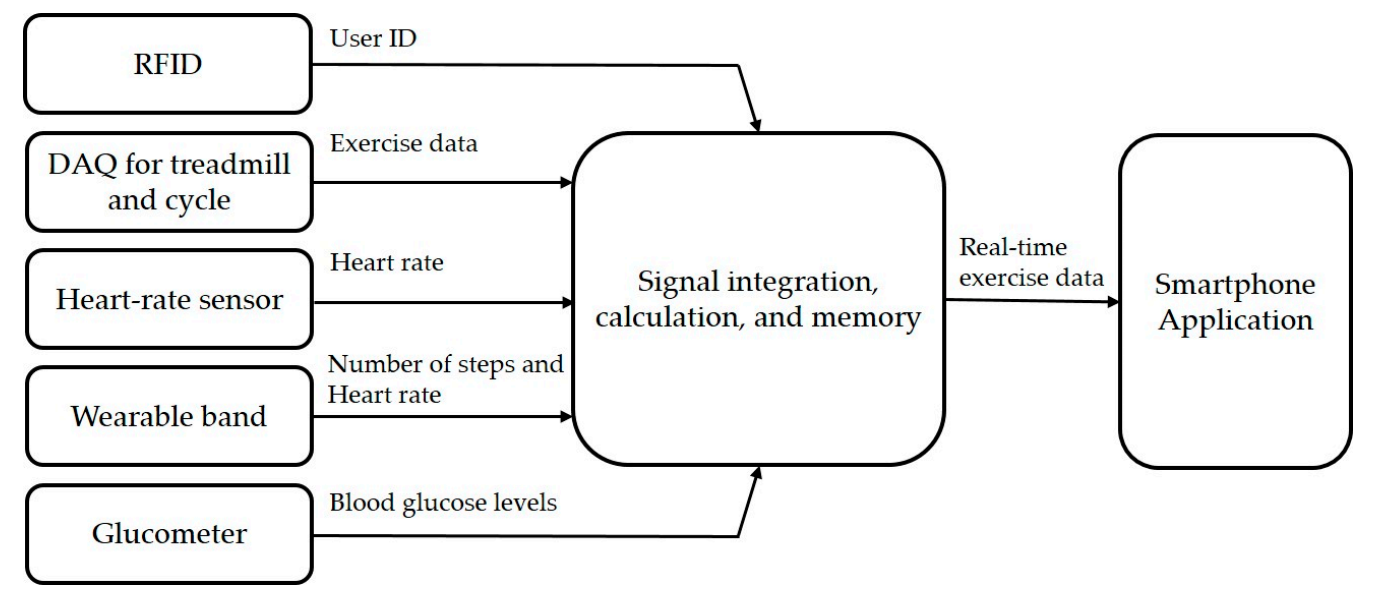

Figure 3. The data flow of the proposed therapeutic exercise platform.

The hardware part of the proposed platform acquires exercise information and heart rate information whenever a patient exercises. The hardware part obtains information by dividing outdoor exercise and indoor exercise.

During exercise, the patients monitor various exercise factors using the proposed hardware configuration. The device configuration differs depending on the outdoor exercise and indoor exercise using a stationary bike and treadmill. When the patients perform jogging or walking outdoors, a wearable band acquires exercise and heart rate information by itself. When exercising indoors, the patient first identifies the user of the exercise equipment using their RFID tag containing their information. Then, they start to exercise using exercise equipment, including DAQ, to obtain the exercise information automatically. They also wear a wearable band or ear-clip heart rate sensor to measure their heart rate.

First, we describe the hardware configuration and operation for outdoor exercise. The proposed platform can monitor outdoor exercise using commercial wearable bands such as Xiaomi's MiBand [35]. They incorporate low-power processors, inertial sensors, and optical biosensors connected to a smartphone through a Bluetooth network. These can measure the number of steps and heart rate and store the obtained data in their memory, with append data synchronization time stored separately. The sum of the magnitudes of the acceleration vectors, $E_{A}$ is calculated as Equation (5).

$$
E_{A}=\left(x^{2}+y^{2}+z^{2}\right)^{0.5}
$$

where $x, y$, and $z$ are signal values along each axis. An interrupt signal is generated when $E_{A}>$ threshold (held in the sensor memory), and the processor then classifies behavior type by interrupt occurrence frequency.

The wearable band starts at the start stage and precedes system initialization as shown in Figure 4. By executing Global variable unit (), System setup (), and Bluetooth setup (), system-wide variables are initialized, processor I/O pins are set, and Bluetooth profiles are set, respectively. Then, the smartphone application initiates information synchronization with the wearable band over a Bluetooth network when it is connected. Next, it compares the data stored by the wearable band and the smartphone application. If the data are matched, it ends immediately. Otherwise, it starts to perform the data synchronization process after device authentication of the wearable band.

In the wearable band, every information is determined through the occurrence time interval when a sensor interrupt signal is generated from the three-axis MEMS (microelectro-mechanical system) accelerometer. If it does not occur, the system switches the 
processor to deep sleep mode to reduce power consumption. The wearable band begins to calculate the number of steps by performing the Data processing () process if the interrupt occurs. Patient physical activity information is automatically transmitted to the smartphone, including walking count, heart rate, and sleeping time. Thus, exercise start time, duration, speed per minute, and end time are derived from the number of steps whenever the patient performs aerobic exercise such as running or walking outdoors. The only exception is a command to measure heart rate. It acts as an external interrupt, Ext.interrupt, and it is transmitted to the wearable band periodically. After this interrupt is received, the wearable band is activated. The acquired heart rate is stored together with time in its internal memory.

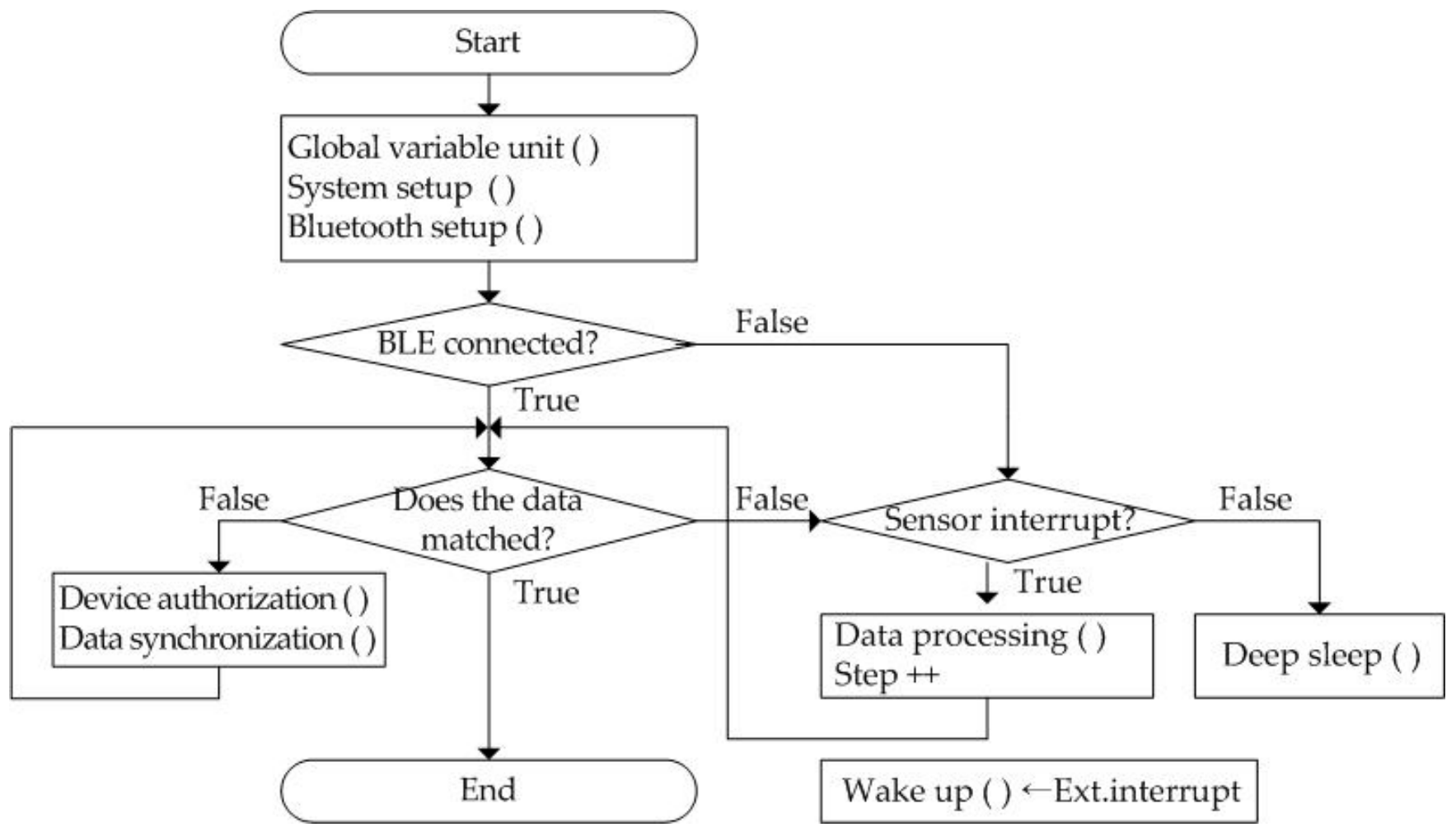

Figure 4. The operation flow of the proposed wearable band.

Second, we use exercise equipment such as stationary bikes and treadmills for indoor exercise. We devised an exercise DAQ board for indoor aerobic exercise equipment to obtain exercise information. The proposed DAQ was linked with a smartphone and wearable band through a Bluetooth network to acquire detailed exercise information, such as exercise start time, duration, end time, exercise load (each minute), and heart rate, in real-time whenever the patient exercised [3]. The mainboard of DAQ is an Intel Curie board, which has a built-in NRF51822 BLE chipset from Nordic [36]. We adopt the magnetic sensor as the SainSmart hall sensor module and the heart rate sensor as the Grove ear-clip heart rate sensor $[37,38]$.

The exercise information acquisition device fabricated in this paper was attached to a treadmill and an ergometer. Table 2 shows the exercise information acquired to help the diabetic patient manage exercise therapy. Exercise start and end times were obtained from the real-time clock module, with average speed, moving distance, exercise load (average heart rate), and calories consumed calculated from the DAQ information. Table 2 shows exercise information that was transmitted in real-time when the smartphone was connected. The data was summarized and automatically stored in the memory unit. 
Table 2. A data packet format for the proposed therapeutic exercise platform.

\begin{tabular}{|c|c|c|c|}
\hline Packet Index & Patient ID & Length of Data & Date \\
\hline Start time & Calories & $\begin{array}{c}\text { Distance } \\
\text { for 1st min. }\end{array}$ & $\begin{array}{l}\text { Average speed } \\
\text { for 1st min. }\end{array}$ \\
\hline $\begin{array}{l}\text { Heart-rate } \\
\text { for 1st min. }\end{array}$ & $\begin{array}{c}\text { Distance } \\
\text { for } 2 \text { nd min. }\end{array}$ & $\begin{array}{l}\text { Average speed } \\
\text { for } 2 \text { nd min. }\end{array}$ & $\begin{array}{l}\text { Heart-rate } \\
\text { for } 2 \text { nd } \min .\end{array}$ \\
\hline$\ldots$ & $\ldots$ & $\ldots$ & $\ldots$ \\
\hline $\begin{array}{c}\text { Distance } \\
\text { for i-th min. }\end{array}$ & $\begin{array}{l}\text { Average speed } \\
\text { for i-th min. }\end{array}$ & $\begin{array}{l}\text { Heart-rate } \\
\text { for i-th min. }\end{array}$ & Maximum heart-rate \\
\hline Average speed & Total exercise time & Total distance & End of Data Packet \\
\hline
\end{tabular}

Exercise amount was obtained from the rotational speed measured by attaching a permanent magnet to the rotating body, as shown in Figure 5 using a hall effect. The processor calculates movement distance, $D_{\text {total }}$, and Instantaneous movement speed, $V_{t}$ as

$$
D_{\text {total }}=C_{\text {dist }} \times N_{\text {rot }}
$$

and

$$
V_{t}=C_{\text {dist }} / \Delta t
$$

where $C_{\text {dist }}$ is a fixed movement distance constant, $N_{\text {rot }}$ is the number of rotation signals, and $\Delta t$ is the time difference between the current time, $t$, and previous time, $t-1$, when the processor recognized the magnetic field monitoring signal from the sensor.

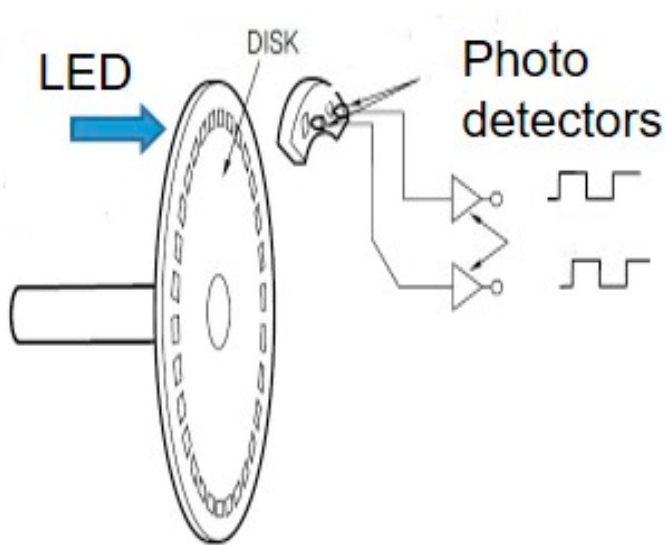

(a) Optical type encoder

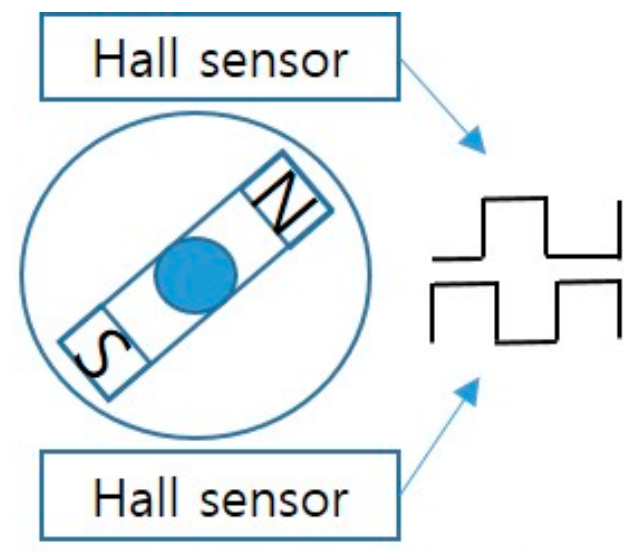

(b) Magnetic type Encoder

Figure 5. Two types of encoder for exercise DAQ device.

Figure 6 shows the motion information acquisition operation. All system variables are initialized when booting starts from the system boot loader. System setup includes peripheral I/O setup, Bluetooth profile setup, memory index processing, and display initialization. Bluetooth connection is checked after initialization completes. The system only measures and stores exercise information if it is not connected, whereas real-time information transmission and synchronization are also performed if it is connected.

The proposed indoor aerobic exercise monitoring method is as follows.

1. The user wears an exercise band or Bluetooth low energy (BLE) based heart rate monitor and starts exercising.

2. The band identifies the user to the exercise equipment through the RFID tag.

3. The proposed exercise monitoring system recognizes exercise commencement when the rotating body starts to rotate and initializes user status and start date, and time. 
4. The proposed DAQ device collects exercise information during exercising: instantaneous exercise speed every second, heart rate every minute.

5. Each exercise equipment stores user identification, date, start time, end time, duration, type, distance, speed per minute, and total distance. It also stores user biometric information, including exercise intensity and heart rate change per minute. The obtained information is transmitted to the smartphone in real-time.

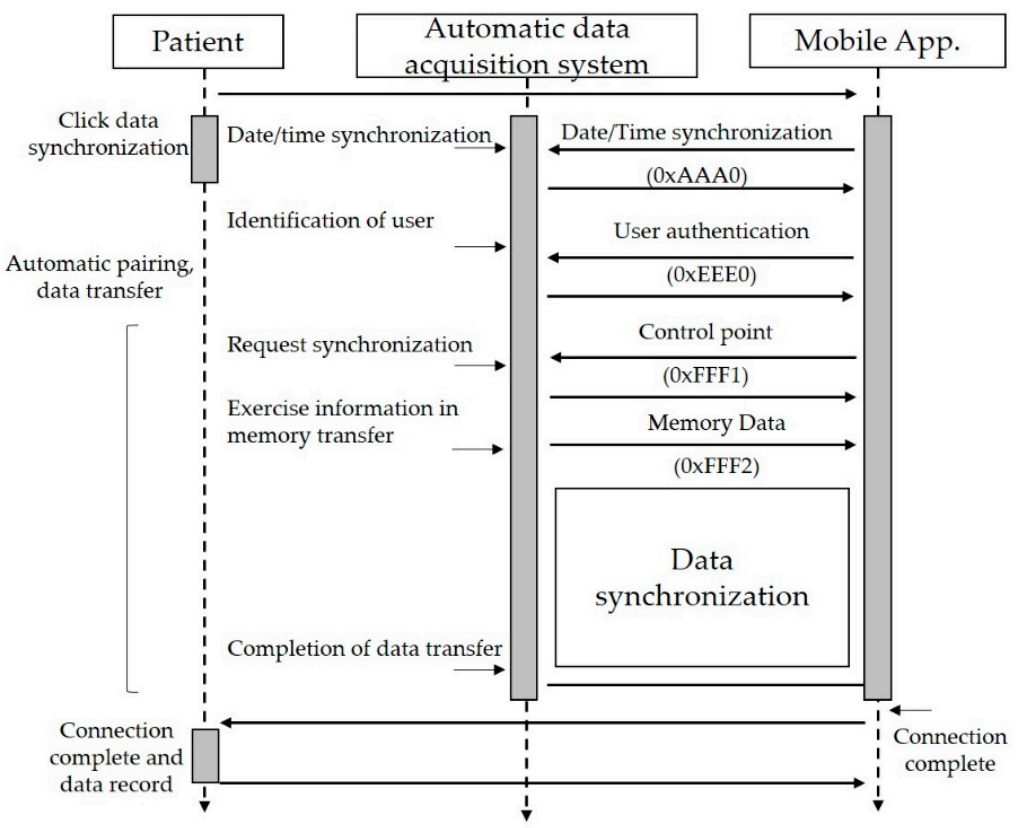

Figure 6. The data synchronization process between aerobic equipment and smartphone application in the proposed therapeutic exercise platform.

\subsection{Simple Exercise Stress Test to Reflect Changes in the Patient's Athletic Ability}

An exercise stress test is often used to diagnose coronary heart disease. In sports medicine, exercise stress testing is presently used to assess physical fitness, determine functional capacity, diagnose cardiac disease, define the prognosis of known cardiac disease, prescribe an exercise plan, and guide cardiac rehabilitation [39-41]. Guidelines on whether or not to perform systematic exercise stress tests for diabetic individuals vary according to the region and professional associations [42]. Riebe et al. stated that preparticipation health screening would be based on the individual's current physical activity level, the desired exercise intensity, the presence of symptoms, and his medical history [41,43]. The American Diabetes Association pointed out pre-exercise medical clearance is unnecessary if the patients want to begin low- or moderate-intensity physical activity not exceeding the demands of brisk walking or everyday living [44,45].

In this section, the proposed platform introduces a simple exercise test that calculates the appropriate amount of exercise for patients during their usual exercise. The proposed exercise test is based on either the Karvonen formula or modified Borg RPE. Patients periodically perform the proposed exercise test to determine their moderate exercise intensity and help them exercise at the right intensity.

In general, the exercise test is performed according to protocols. The most common protocol used during the exercise test is the Bruce protocol. The test is performed in a designated lab, supervised by trained healthcare personnel. The patient closed their eyes for 5 minutes, and then their heart rate and blood pressure were measured at rest. The exercise test was terminated if the patient had experienced any subjective symptoms (difficulty breathing, local fatigue, leg pain) or maximum blood pressure increased by $250 / 115 \mathrm{mmHg}$ (millimeters of Mercury) or more. The test used a treadmill, electrocardiogram (ECG), metabolic cart, blood pressure monitor, perceived exertion, and pulse meter. The modified 
Bruce protocol is preferred for exercise stress testing, as shown in Table 3 [46]. Its outcomes are well-validated, and exercise capacity measured in MET has a good prognostic value. Kim et al. [47] presented the study to provide valuable data for CR-10 as the RPE. Using these results, we reconstructed as shown in Table 3 as an exercise load test scale for the proposed platform. The test started at low intensity and gradually increased intensity for each step, as shown in Table 3.

Table 3. Modified treadmill protocol for exercise stress testing for the proposed therapeutic exercise platform.

\begin{tabular}{|c|c|c|c|c|c|}
\hline \multirow{2}{*}{$\begin{array}{c}\text { Protocol } \\
\text { Stage (3 min.) }\end{array}$} & \multicolumn{3}{|c|}{ Modified Bruce Protocol } & \multirow{2}{*}{$\begin{array}{l}\text { Estimated } \\
\% \text { HRR }\end{array}$} & \multirow{2}{*}{$\begin{array}{l}\text { Estimated } \\
\text { CR-10 RPE }\end{array}$} \\
\hline & Speed & Grade & MET & & \\
\hline Stage 1 & $\begin{array}{l}1.7 \mathrm{mile} / \mathrm{h} \\
(2.7 \mathrm{~km} / \mathrm{h})\end{array}$ & $0 \%$ & 2 & - & - \\
\hline Stage 2 & $\begin{array}{l}1.7 \mathrm{mile} / \mathrm{h} \\
(2.7 \mathrm{~km} / \mathrm{h})\end{array}$ & $5 \%$ & 3 & - & - \\
\hline Stage 3 & $\begin{array}{l}1.7 \mathrm{mile} / \mathrm{h} \\
(2.7 \mathrm{~km} / \mathrm{h})\end{array}$ & $10 \%$ & 4 & $58 \%$ & 1.48 \\
\hline Stage 4 & $\begin{array}{l}2.5 \mathrm{mile} / \mathrm{h} \\
(4.0 \mathrm{~km} / \mathrm{h})\end{array}$ & $12 \%$ & 7 & $68 \%$ & 2.80 \\
\hline Stage 5 & $\begin{array}{l}3.4 \mathrm{mile} / \mathrm{h} \\
(5.4 \mathrm{~km} / \mathrm{h})\end{array}$ & $14 \%$ & 10 & $84 \%$ & 4.55 \\
\hline Stage 6 & $\begin{array}{l}4.2 \mathrm{mile} / \mathrm{h} \\
(6.7 \mathrm{~km} / \mathrm{h})\end{array}$ & $16 \%$ & 13 & $95 \%$ & 6.70 \\
\hline
\end{tabular}

Borg developed a RPE $[48,49]$. It is a widely used and reliable indicator to monitor and guide exercise intensity by allowing patients to rate their level of exertion during exercise subjectively. Two RPE scales are commonly used; the original Borg scale (6 to 20 scale) and the revised category-rate scale, CR-10 ( 0 to 10 scale). A high correlation exists between a person's perceived exertion rating times ten and the actual heart rate during physical activity, so a person's exertion rating may provide a relatively good estimate of the actual heart rate during activity [45]. In the proposed therapeutic exercise platform, we used the CR-10 Borg scale, not the original Borg scale, increasing exercise awareness between 1 3 MET each step. The other most significant advantage of the CR-10 is its easy handling by allowing daily changes in the exercise.

Most patients undergo exercise stress tests in hospitals at the initial diagnosis of diabetes, and they receive appropriate exercise prescriptions. As patients repeat the same intensity exercise for a long time, they feel the exercise intensity gradually lower. Besides, the patient's physical strength varies depending on several factors. To maintain the prescribed intensity of exercise, patients should repeatedly check their exercise stress during their usual exercise. The proposed therapeutic exercise platform supports a simple exercise stress test to check the effect of exercise intensity on heart rate and RPE. It adjusts the exercise intensity according to the results and informs the patient about the adjusted exercise intensity to continue exercising according to the exercise prescription.

Figure 7 shows an example of an exercise prescription at the early stages of diabetic diagnosis. After the exercise test, the patient receives the prescribed target heart rate and weekly target exercise intensity based on heart rate, as shown in Figure 7a. Figure 7b shows a look-up table. During the exercise test, the patient's heart rate and the RPE are linked with the exercise intensity at each test step, and they are recorded in a look-up table. This table shows the relationship between the heart rate and exercise speed obtained each time the patient exercised. It is used to tune the exercise speed in the exercise session before the patient exercises.

The proposed exercise test is based on the modified Bruce protocol, and it is conducted for about 20 minutes at the exercise intensity shown in Table 3 using a treadmill or a stationary bike. The patient maintains a low exercise intensity for 9 mins. For the remaining 15 mins, the intensity is increased gradually to reach the target heart rate. During the test, 
the patient's heart rate, exercise intensity, and RPE are obtained in real-time. A wearable band or ear-clip heart rate sensor is to obtain the heart rate. Exercise intensity and RPE are obtained from the proposed DAQ and chatbot, respectively.

\begin{tabular}{|c|c|c|c|c|}
\hline $\begin{array}{l}\text { - } \quad \text { Targ } \\
\text { - } \quad 6 \text { we }\end{array}$ & $\begin{array}{l}\text { Exercise } \\
\text { et heart rate, } \\
\text { ek prescribing }\end{array}$ & $\begin{array}{l}\text { pres } \\
\text { THR : } \\
\text { reha }\end{array}$ & $\begin{array}{l}\text { cription } \\
\mathrm{A}_{3}-\mathrm{A}_{4} \mathrm{bpm} \\
\text { b program }\end{array}$ & \\
\hline Weeks & Low intensity & $\%$ & High intensity & $\%$ \\
\hline 1-2 week & $\mathrm{A}_{1} \mathrm{bpm}(40 \%)$ & $80 \%$ & $\mathrm{~A}_{2} \mathrm{bpm}(50 \%)$ & $20 \%$ \\
\hline 3-4 week & $\mathrm{A}_{2} \mathrm{bpm}(50 \%)$ & $80 \%$ & $\mathrm{~A}_{3} \mathrm{bpm}(60 \%)$ & $20 \%$ \\
\hline 5-6 week & $\mathrm{A}_{3} \mathrm{bpm}(60 \%)$ & $80 \%$ & $\mathrm{~A}_{4} \mathrm{bpm}(70 \%)$ & $20 \%$ \\
\hline
\end{tabular}

(a)

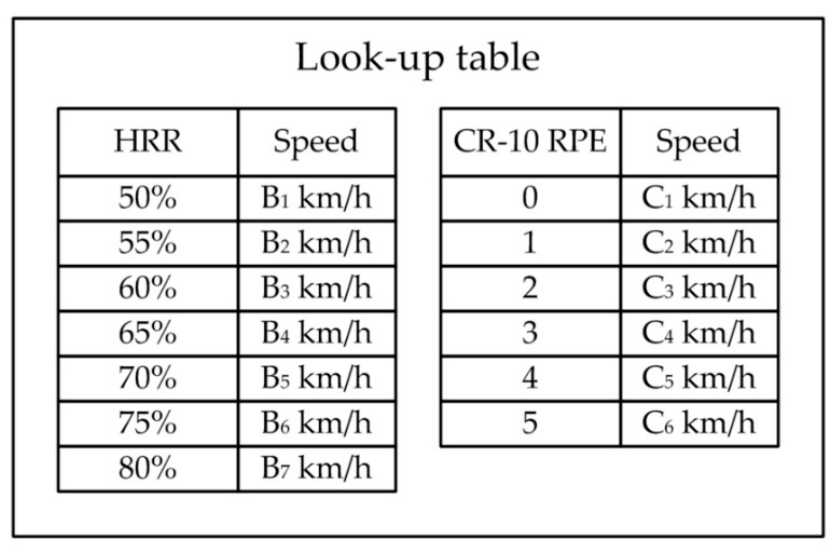

(b)

Figure 7. User input information to set the proposed therapeutic exercise platform; (a) Example of exercise prescription based on the exercise stress test, (b) look-up table to calculate exercise intensity based on HRR and CR-10 RPE.

The patient performs the proposed test by selecting one of the following three methods depending on the available exercise equipment and vital sensors. The first method is based on the modified Bruce protocol, and it is used when the patient has a treadmill suitable for the protocol. The second method is used when the user exercises using a treadmill or indoor bike with a heart rate sensor. The third method is used when the user exercises outdoors or wants to test with RPE with a wearable band. In all cases, patients should measure their resting heart rate, $H R_{\text {rest }}$ before the exercise test.

The first method performs an exercise test according to the modified Bruce protocol shown in Table 3. At each stage, the patient's heart rate is measured, and the CR-10 RPE felt by the patient is also recorded. According to the heart rate measured in each stage, the speed and grade are determined as the recommended exercise intensity.

The second method is to perform an exercise test by simply measuring the heart rate in real-time. As shown in Table 3, the patient starts the exercise from the low intensity according to the exercise protocol using a treadmill or a stationary cycle. They perform the exercise for 9 mins when it reaches $58 \%$ of the heart rate reserve. Then, the patient increases the exercise intensity until the target heart rate is reached, as shown in Table 3. During the proposed exercise test, the measured heart rate is displayed to the patient. After the examination, the exercise speed of the treadmill or cycle for each stage is recommended as the patient's exercise intensity.

The last method is to perform a simple exercise test with a modified Borg RPE that the patient feels himself. The patient maintains low-intensity exercise on a treadmill or bike for 9 mins before reaching level 1.48 of the modified Borg RPE. As shown in Table 3, the patient increases the exercise speed until the target RPE level is reached and maintains the exercise speed for 3 minutes. The recommended exercise intensity is determined by the average exercise speed of each stage of each RPE recorded.

The proposed test allows the patients to run simply exercise tests whenever necessary. The test result is used to adjust the intensity of exercise to match the patient's exercise ability. Busch et al. [50] presented the classification of physical activity intensity using the 2009 ACSM (American College of Sports Medicine) classifications. Low intensity is classified as $50-63 \% H R R_{\max }$. Moderate and vigorous intensities are $64-76 \% H R R_{\max }$ and $77-93 \%$ $H R R_{\text {max }}$, respectively. We considered the appropriate exercise intensity to be sufficient to reach $50-76 \% H R R_{\max }$. Additionally, moderate and intermittent medium or high-intensity exercise for $45 \mathrm{~min}$ or more is beneficial for blood glucose management. Consequently, patients can adjust their exercise intensity and time after setting the coaching algorithm 
according to their abilities. We developed a smartphone application to provide an exercise monitoring and coaching program based on their exercise stress test results.

\subsection{Proposed Real-Time Exercise Monitoring Application}

For diabetics, exercise therapy is vital for blood glucose management. However, diabetic patients are less aware of the importance of exercise therapy due to the fear of hypoglycemia caused by excessive exercise and the different physical abilities [4]. This section describes the proposed exercise monitoring and coaching method to inform the patient of the appropriate exercise intensity and exercise time using the proposed therapeutic exercise platform. In this section, exercise intensity refers to the exercise speed according to the patient's heart rate during exercise.

The proposed exercise monitoring and coaching application help diabetics continue to exercise according to a prescribed exercise protocol. First, the patients launch the coaching application, and they enter the type of exercise and the scheduled exercise time. The coaching application recommends an exercise plan that combines intermediate and intermittent high-intensity exercises according to the exercise test results in advance.

For example, each exercise coaching instance consists of a set of $45 \mathrm{~min}$. The patient exercises according to a predetermined exercise coaching algorithm. This application measures the patient's heart rate every minute during exercise and guides them to maintain the recommended exercise speed. The proposed application alerts the patient in real-time if the patient's exercise speed is too low or too high (e.g., if the patient's heart rate is below $50 \%$ of HRmax or above $75 \%$ of HRmax). It also sends a warning if the patient does not exercise at the recommended exercise speed. After completing an exercise, the collected exercise information is summarized, and statistics are generated.

The data acquisition of the proposed platform supports not only automatic input from sensors, but also voice or manual input by the user as shown in Figure 8. The proposed application allows the user to voice input of RPE values using a chatbot during exercise. In addition, the user can manually modify the record when not exercising. The information collected during the patient's exercise fine-tunes the exercise program to the patient's exercise goal. The real-time monitoring of the proposed application has four display modes. The first mode is an exercise plan mode. If there is an exercise prescription, a recommended exercise plan is displayed. Otherwise, it displays the exercise mode selected by the user. The remaining three modes show the comparison result of the recommended exercise and the exercise being performed for exercise speed, heart rate, and RPE, as shown in Figure 8.

\section{Hardware platform Therapeutic exercise application Monitoring UI}

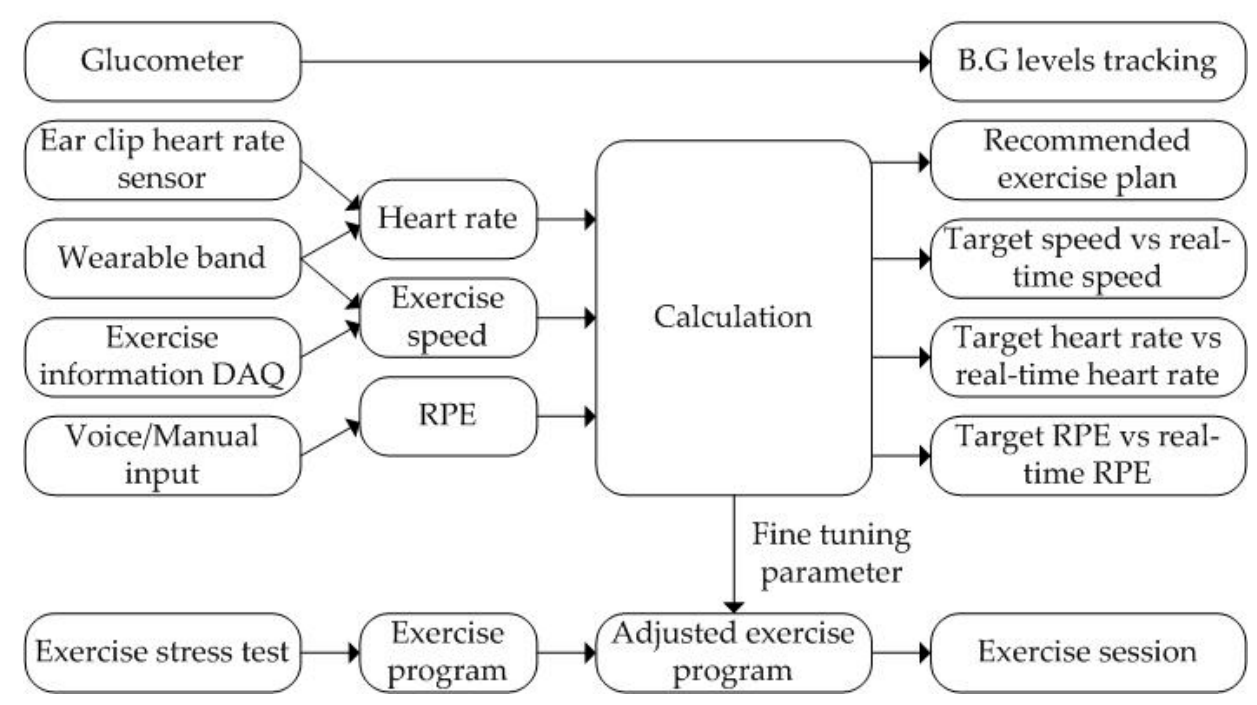

Figure 8. The proposed therapeutic exercise platform for an exercise coaching program. 


\section{Evaluation Results}

We devised the following three hardware configurations to acquire exercise data and vital signs during exercise. First, the proposed platform used Xiaomi's Mi band, a commercial wearable band, to acquire the number of steps and heart rate by time. Second, an exercise DAQ device was added to the treadmill and stationary bike to obtain the exercise information. Third, an ear-clip heart rate sensor was added to obtain the heart rate during exercise. These were linked with a smartphone application through BLE communication with a glucometer.

The exercise DAQ was mounted on the aerobic exercise device, and it included a magnetic sensor, SD card, and BLE module in a 32-bit processor. We chose an Intel Curie board as microcontroller unit because it was also easy to use and compatible with Genuino and Arduino 101. It connected with an external RFID unit and a heart rate sensor to obtain user identification and heart rate information.

The first experiment was to evaluate the accuracy of the exercise speed information acquired through DAQ. As shown in Tables 4 and 5, the rotation axis of two stationary bikes (A, B) and two treadmills (A, B) was rotated 1,000 times at different speeds and then obtained from DAQ. Both experiments had less than $2 \%$ error and accuracy close to $100 \%$ at low speeds. The results indicate that the exercise speed obtained from the designed DAQ had an accuracy of at least $98 \%$.

Table 4. Experiment result for calculating the exercise speed of an indoor cycle.

\begin{tabular}{ccccccc}
\hline \multirow{2}{*}{ Item } & \multicolumn{5}{c}{ Speed of an Indoor Cycle } \\
\cline { 2 - 7 } & $\mathbf{1 0} \mathbf{~ k m} / \mathbf{h}$ & $\mathbf{1 5} \mathbf{~ k m} / \mathbf{h}$ & $\mathbf{2 0} \mathbf{~ k m} / \mathbf{h}$ & $\mathbf{2 5} \mathbf{~ k m} / \mathbf{h}$ & $\mathbf{3 0} \mathbf{~ k m} / \mathbf{h}$ & $\mathbf{3 5} \mathbf{~ k m} / \mathbf{h}$ \\
\hline 1000 turns (A) & 1000 & 1000 & 999 & 998 & 995 & 990 \\
1000 turns (B) & 1000 & 1000 & 998 & 999 & 996 & 991 \\
Accuracy & $100 \%$ & $100 \%$ & $99.9 \%$ & $99.8 \%$ & $99.5 \%$ & $99.1 \%$ \\
\hline
\end{tabular}

Table 5. Experiment result for calculating the exercise speed of a treadmill.

\begin{tabular}{|c|c|c|c|c|c|c|}
\hline \multirow{2}{*}{ Item } & \multicolumn{6}{|c|}{ Speed of a Treadmill } \\
\hline & $4 \mathrm{~km} / \mathrm{h}$ & $5 \mathrm{~km} / \mathrm{h}$ & $6 \mathrm{~km} / \mathrm{h}$ & $7 \mathrm{~km} / \mathrm{h}$ & $8 \mathrm{~km} / \mathrm{h}$ & $9 \mathrm{~km} / \mathrm{h}$ \\
\hline 1000 turns (A) & 1000 & 1000 & 999 & 1000 & 998 & 980 \\
\hline 1000 turns $(\mathrm{B})$ & 1000 & 1000 & 1000 & 1000 & 998 & 982 \\
\hline Accuracy & $100 \%$ & $100 \%$ & $99.9 \%$ & $100 \%$ & $99.8 \%$ & $98.1 \%$ \\
\hline
\end{tabular}

The second experiment was to measure the accuracy of the heart rate acquired from the heart rate sensor. During exercise using a stationary bike and a treadmill, the heart rate obtained from DAQ and the result of Polar's T34 heart rate monitor were compared. The result is shown in Figure 9. The measurement error was up to $2 \%$, and the average error was within $1 \%$, indicating that an error rate is irrespective of the movement speed. Therefore, the proposed DAQ could measure real-time heart rate with at least $98 \%$ accuracy regardless of changes in exercise intensity.

After we evaluated the proposed therapeutic exercise platform, including the hardware part and the smartphone application, we tested the accuracy in an actual exercise environment. The comparison results of the proposed DAQ and a commercial heart rate monitor, Polar T34, are shown in Figure 10. The exercise was performed in the order of warm-up, intermittent high intensity, and medium intensity. Figure 10 shows the patient's heart rate change according to the change in exercise intensity. In the warm-up phase, the heart rate gradually increased, and in the high-intensity exercise section, the heart rate increased to $80 \%$ of the maximum heart rate. After that, it indicates that the heart rate stably lowered in the middle-intensity exercise section. The average difference is within $2 \%$. This result proves that the proposed therapeutic exercise platform was suitable for monitoring exercise in real-time. 


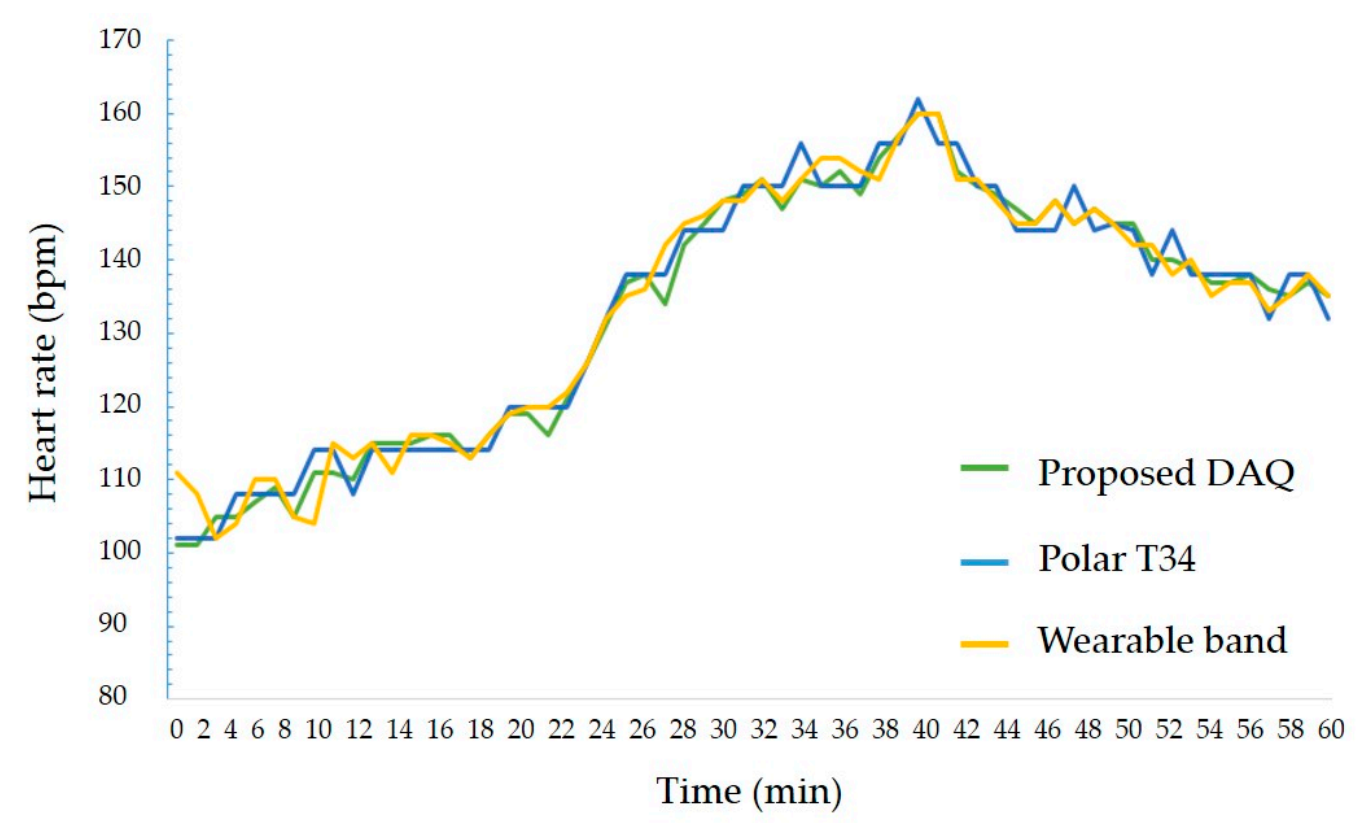

Figure 9. The experiment result of heart rate sensing between proposed DAQ and other counterparts.

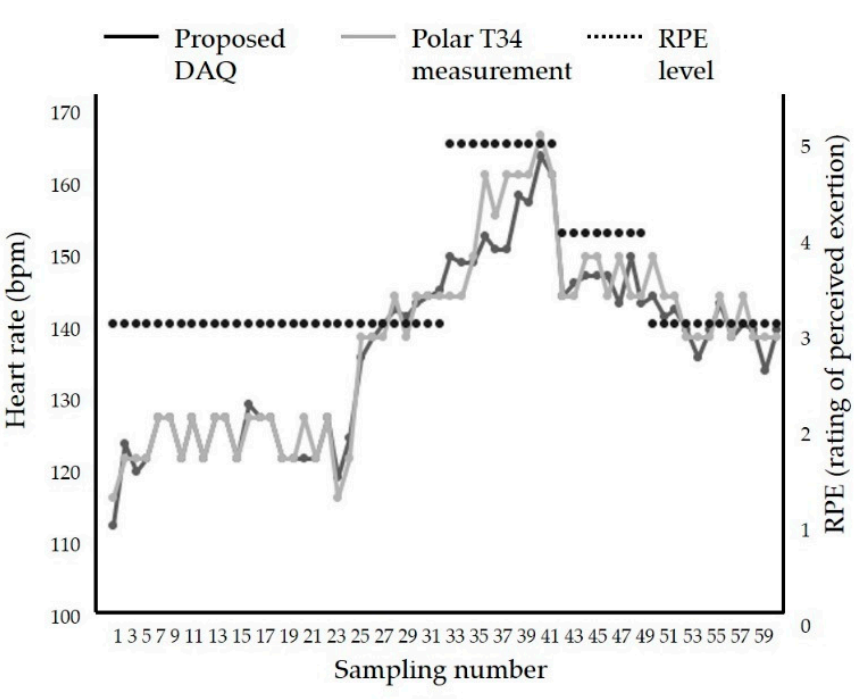

(A)

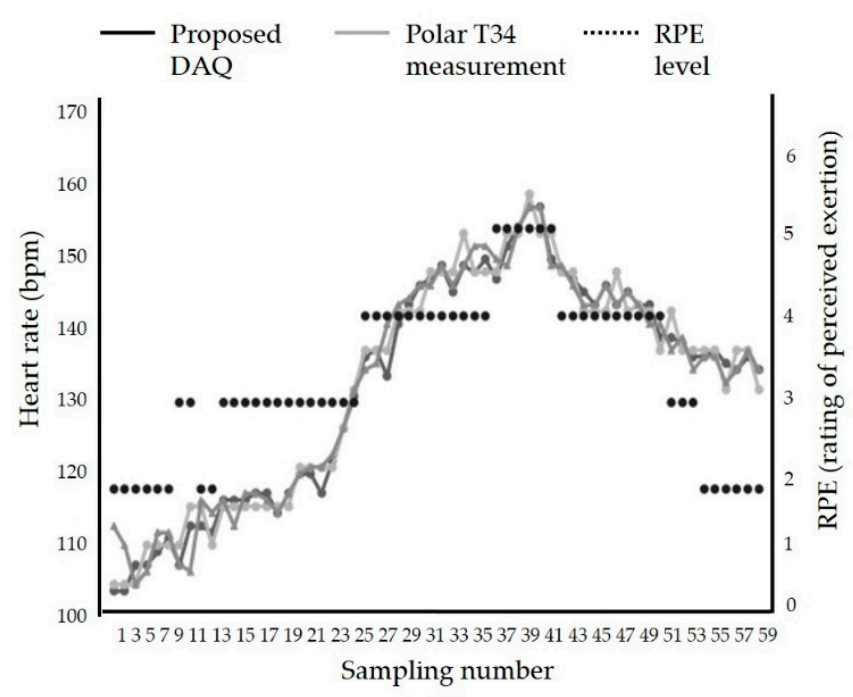

(B)

Figure 10. Comparison results between the heart rate during exercise using; (A) Treadmill, (B) stationary bike.

The proposed therapeutic exercise application was implemented using two languages, Java 1.8 and Kotlin [51]. The smartphone operating system was set to Android. The minimum version of the supported Android operating system was set to API (application programming interface) 19 (version name: Kitkat) that supports low-power Bluetooth. The proposed application was implemented as shown in Figure 11. It consisted of the initial screen, entered user information, device registration, and management. Furthermore, the patients input their exercise prescription or exercise setting determined by patients. Then, the application could help the patients to exercise their prescription or exercise plan.

Figure 12 shows the results of exercise monitoring. First, the patient could perform a simple exercise test. The proposed simple exercise load test could be performed by selecting one of the modified Bruce protocol, HRR, and RPE modes. The test results were used to determine the appropriate exercise intensity for the patient, as shown in Figure 12a. During exercise, the patient can monitor in real-time whether he exercises according to the exercise plan. In this case, the patient could select monitoring mode three modes of exercise speed, heart rate, or RPE. Figure $12 \mathrm{~b}$ is a real-time monitoring screen showing 
whether the patient was exercising according to the exercise plan based on the heart rate. After the exercise is over, the exercise result was summarized and displayed to the patient, as shown in Figure 12c.

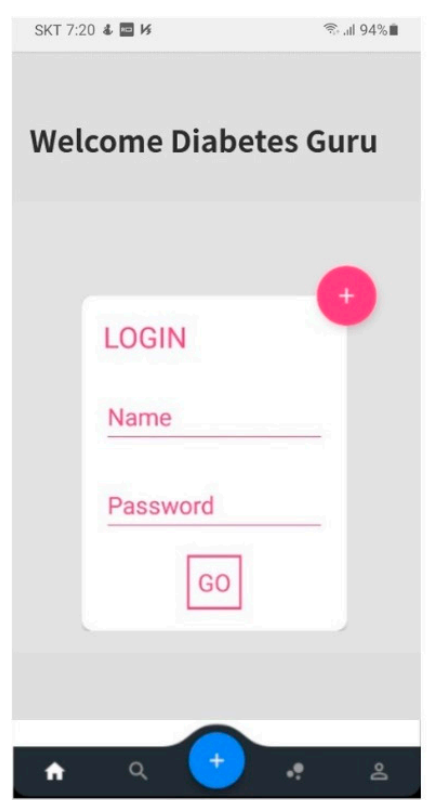

(a)

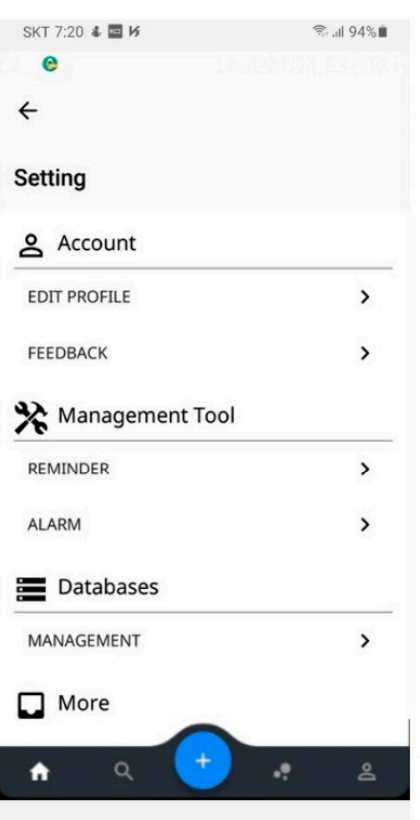

(b)

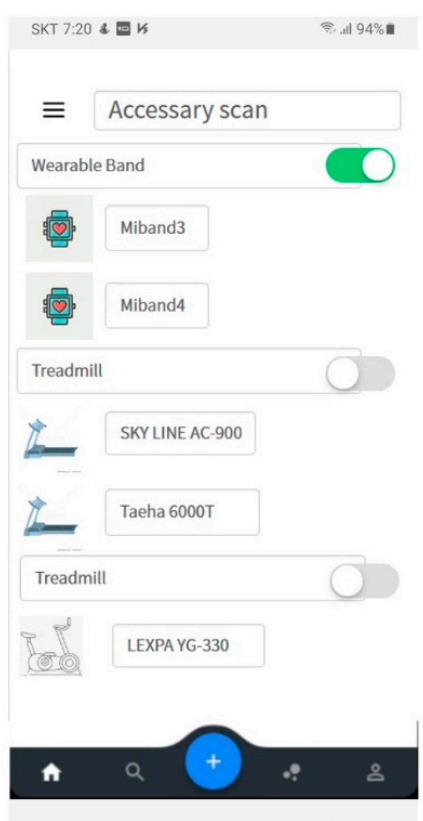

(c)

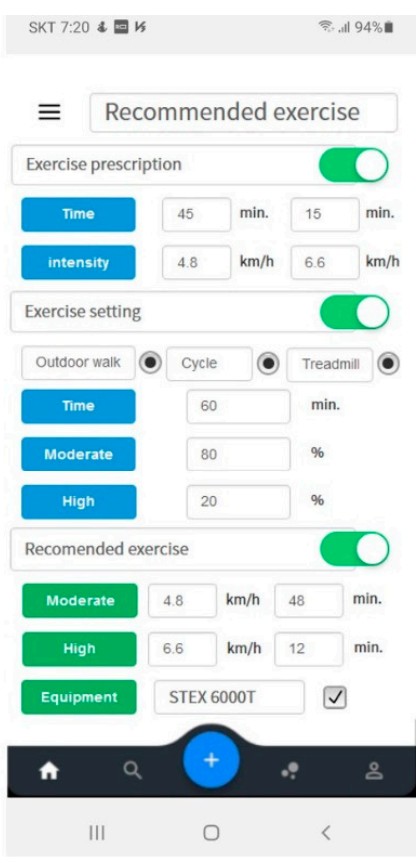

(d)

Figure 11. Proposed mobile application user interface (UI); (a) log-in, (b) application setting, (c) device scan, (d) input of exercise prescription or user's plan and recommended exercise plan.

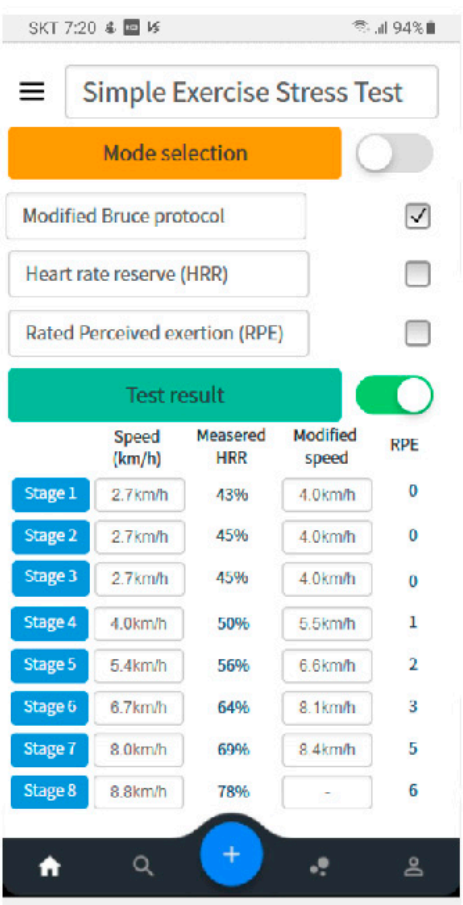

(a)

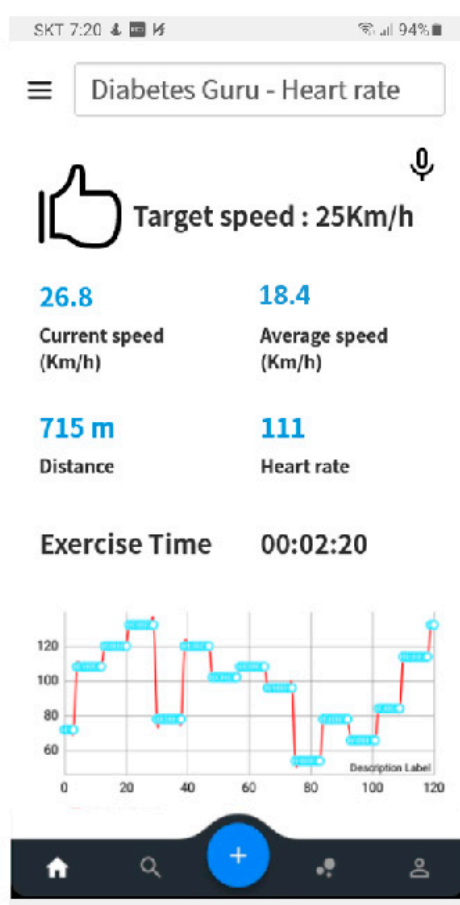

(b)

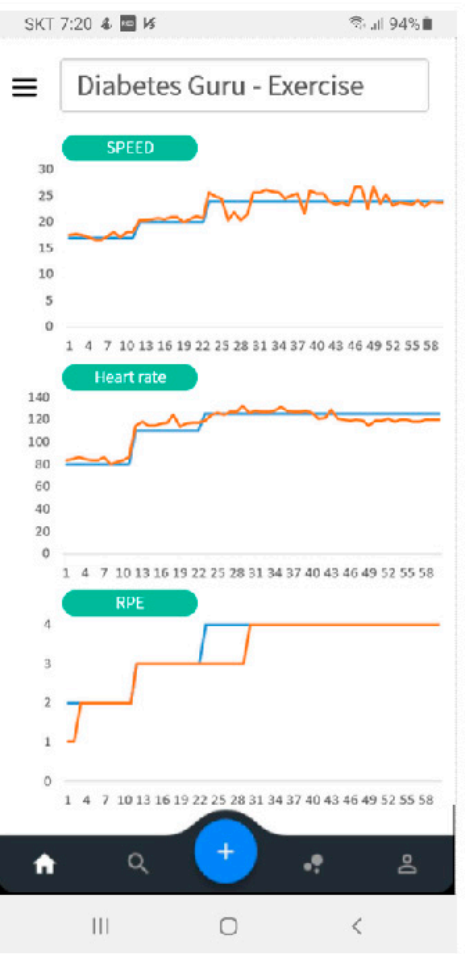

(c)

Figure 12. User interface configuration of proposed therapeutic exercise application; (a) Results of simple exercise stress test, (b) real-time monitoring based on heart rate, (c) exercise summary after exercise finish. 
Table 6 shows the comparison results between the proposed therapeutic exercise platform and other counterparts. Automatic refers to the ability of an application to connect with a device and automatically record relevant data to the application. Manual represents that the user directly enters the relevant records into the application. Additionally, exercise plan adjust refers to a function of automatically adjusting an exercise program according to a user's exercise experience. The significate feature is that the proposed platform was linked with aerobic exercise equipment, a wearable band, and a heart-rate sensor. The platform gathered precise exercise information and heart rate during exercise. It used the information to adjust the exercise program of the exercise session to keep the excise intensity indicated from the exercise prescription. An exercise stress test in the proposed therapeutic exercise platform could estimate the relationship between the patient's heart rate and exercise speed. If the patients keep doing aerobic exercise for a long time, it is good to improve their physical fitness and exercise ability. Therefore, the intensity and loads should continually increase over time. Whenever a patient felt that his or her exercise ability improved, an exercise stress test was performed to obtain a relationship between maximum heart rate reserve and exercise speed. The proposed platform adjusted the exercise intensity in the exercise session by reflecting the exercise stress test results. The exercise speed of the exercise session was adjusted to match the maximum heart rate reserve of the exercise plan. Patients could exercise by adjusting the exercise intensity according to their physical changes.

Table 6. Comparison results between the proposed application and the conventional counterparts.

\begin{tabular}{ccccc}
\hline \multirow{2}{*}{ Index } & Name & BG Levels & Steps & Exercise \\
\hline \multirow{3}{*}{ Researches } & Jeon et al. [52] & Automatic & - & Manual \\
\cline { 2 - 5 } & Kim et al. [53] & Automatic & Automatic & Manual \\
\cline { 2 - 5 } & Årsand et al. [54] & Automatic & Automatic & Manual \\
\cline { 2 - 5 } & Osbone et al. [55] & Automatic & Automatic & Manual \\
\cline { 2 - 5 } Commercial App. & Diabetes: M [56] & Automatic & - & - \\
\cline { 2 - 5 } & WISHCARE [57] & Automatic & Automatic & - \\
\cline { 2 - 5 } & iCareD [58] & Automatic & Automatic & Manual \\
\cline { 2 - 5 } & MGHelthNote [59] & - & Automatic & Automatic \\
\hline Proposed App. & Monitor Diabetes [60] & Manual & Manual & Manual \\
\hline
\end{tabular}

A closed beta service has been performed since 2017. We released our application to Google play store for a closed test. Among the users who used the application who answered that they had a high understanding of diabetes, diabetic patients and those who did not have a diagnosis of diabetes were selected and the experimental group was recruited. Finally, we selected the patient population from patients with a prevalence of 3 years or more and a fasting blood glucose level greater than $160 \mathrm{mg} / \mathrm{dL}$. The questionnaire is shown in Figure 13. For platform evaluation and future improvement, we received satisfaction ratings and functional improvements through a questionnaire survey as shown in Figure 14 on 10 type 2 diabetes patients and ten persons without a diabetes diagnosis as shown in Table 7. Overall, the patients and others expressed high satisfaction with ease of use. The patient's additional opinions are as follows. First, it is necessary to update diabetes education about therapeutic exercise. Second, exercises after a meal increased compared to before use. Third, if the function to share experiences with other users is added, motivation will be better. Fourth, it is necessary to provide the personal exercise coaching or consulting function for an exercise. Finally, the number of symptoms of hypoglycemia decreased after exercise. Moreover, it became possible to control the exercise time and intensity according to the amount of meal. 
Questions about recruiting the experimental group are:

(In Korea, regular health check-ups are performed every 1 or 2 years for all workers. Diabetes check-ups are included in the health check-up results)

1 . What is your age?

2. What is your gender?

3. What is your education level?

4. Respond to the results of a recent medical exam, or hospital diagnosis related to diabetes

(1) Normal (2) Prediabetes or suspected diabetes (3) Diabetes (4) I don't know

5. If you have been diagnosed with diabetes, please answer the following additional questions:

(1) Do you have type 1 diabetes? Do you have type 2 diabetes?

(1) Type 1 diabetes (2) Type 2 diabetes (3) Others (4) I don't know

(2) When were you diagnosed with diabetes?

(3) What is your fasting blood sugar during the test?

(4) Did you receive diabetes education at the hospital?

(1) Yes (2) No (3) I don't know

(5) Did you have an exercise test at the hospital?

(1) Yes (2) No (3) I don't know

(6) What is your fasting blood sugar levels?

Figure 13. Questions about recruiting the experimental group.

Questions about recruiting the experimental group are:

1. This is a subjective questionnaire. Please write your comments.

(1) How many times a week do you exercise?

(2) How much time do you exercise each week?

(3) Has the exercise time changed after using the application? If it has changed, please write the number of hours per week changed in detail.

(4) When you performed the exercise suggested by the program, did you feel any physical discomfort?

(5) Did you exercise with the suggested exercise intensity?

(6) If the suggested exercise intensity was not followed, what was the reason?

(7) Please write down the items that need improvement.

2. Please select from 1 to 5 for the following questionnaire (patient group and control group)

(1: Very dissatisfied, 2: Dissatisfied, 3: Average, 4: Satisfied, 5: Very satisfied)

(1) Is this application convenient to use?

(2) Do you use this app for every workout?

(3) Are you satisfied with the UI when exercising?

(4) Does this app help me keep exercising?

(5) Can you predict the exact exercise time and exercise intensity before exercise?

(6) Do you feel your personal information is protected?

(7) Has your fasting blood sugar decreased?

(8) Do you think it will help you maintain your target blood sugar level?

3. Please select from 1 to 5 for the following questionnaire (patient group)

(1: Very dissatisfied, 2: Dissatisfied, 3: Average, 4: Satisfied, 5: Very satisfied)

(1) Has the number of blood glucose measurements increased after using this app?

(2) Do you measure blood sugar before and after exercise?

(3) Is the exercise intensity recommended by this app appropriate?

(4) Has hypoglycemia or hyperglycemia decreased after using this app?

Figure 14. Questions for users to rate the application.

Table 7. Survey results for characteristics of the proposed self-management IoT platform.

\begin{tabular}{|c|c|c|c|c|c|c|}
\hline Index & Survey Content & 5 & 4 & 3 & 2 & 1 \\
\hline Total & Easy to use & $90 \%$ & $10 \%$ & - & - & - \\
\hline \multirow{3}{*}{ Efficiency } & Excellence in exercise monitoring & $80 \%$ & $10 \%$ & $10 \%$ & - & - \\
\hline & Motivation to enable continuous exercise & $80 \%$ & $20 \%$ & - & - & - \\
\hline & Estimating the correct amount of exercise & $70 \%$ & $20 \%$ & $10 \%$ & - & - \\
\hline \multirow{3}{*}{ Safety } & Increase of BG level check pre-or post-exercise & $90 \%$ & - & $10 \%$ & - & - \\
\hline & Suitability of the recommended exercise & $70 \%$ & $10 \%$ & $10 \%$ & $10 \%$ & - \\
\hline & Reduction in hypo- or hyperglycemia & $70 \%$ & $20 \%$ & $10 \%$ & - & - \\
\hline Security & Secure personal data management & $90 \%$ & - & $10 \%$ & - & - \\
\hline
\end{tabular}




\section{Conclusions}

In this paper, we present a therapeutic exercise platform. The proposed platform can support diabetic patients in managing their blood glucose levels by balancing physical exercise, insulin dosage or medication, blood glucose monitoring, and diet. In particular, well-planned and structured exercise is vital to control blood glucose levels in diabetes and reduce the risk of developing diabetes complications.

The proposed system has three main functions. First, it automatically obtains exercise information precisely whenever patients exercise outdoors or indoors. Second, it can analyze the change of exercise ability due to the long-term exercise regime, i.e., a simple exercise test that frequently allows the user to test their athletic ability. Third, it provides an exercise monitoring and coaching program that informs the user whether they are exercising appropriately during a session. Therefore, most patients consider exercise as a supplemental glycemic control method with medication or diet. The proposed therapeutic exercise platform is crucial to develop a therapeutic exercise program that can recommend an exercise suitable for glycemic control to patients according to their preferred exercise pattern and individual exercise ability.

The proposed therapeutic exercise platform consists of a hardware part responsible for obtaining exercise and heart-rate data and smartphone applications that provide a simple exercise test and real-time exercise monitoring results. The proposed exercise test is to calculate the appropriate amount of exercise for the patient. The proposed test can be performed not only by the typical modified Bruce protocol but also by the method based on heart rate reserve and rated perceived exertion for convenience. The evaluation result shows that the exercise speed and heart rate in the proposed hardware part have less than $2 \%$ error. This fact proves that the proposed DAQ can be applicable for various exercise environments regardless of exercise intensity changes. Ten diabetic patients and ten person without a diabetes diagnosis used the proposed platform for more than three months, and we analyzed the effectiveness of the proposed platform through their survey. Overall, the patients expressed high satisfaction with ease of use. Besides, most of the patients reported that both the number of blood glucose measurements and the exercise increased. This study conducted a usability evaluation of the proposed exercise platform with limited users only. Future studies should aim to address the limitations that exist in our work. We plan to verify the functionality of the blood glucose management platform, including exercise therapy, by accumulating pre- and post-exercise blood glucose levels for a long period of time for diabetic patients. Consequently, the proposed therapeutic exercise platform can be useful for the patients to make a well-planned and structured exercise for their glycemic control.

Author Contributions: Conceptualization, J.-H.L.; methodology, J.-H.L.; software, J.-C.P. and J.-H.L.; validation, S.-B.K. and J.-H.L.; formal analysis, J.-C.P. and J.-H.L.; investigation, J.-C.P. and J.-H.L.; resources, J.-H.L.; data curation, J.-H.L.; writing-original draft preparation, J.-C.P. and J.-H.L.; writing-review and editing, S.-B.K. and J.-H.L.; visualization, S.-B.K. and J.-H.L.; supervision, J.H.L.; project administration, J.-H.L.; funding acquisition, J.-H.L. All authors have read and agreed to the published version of the manuscript.

Funding: This research was supported by National Research Foundation of Korea (NRF-2017R1 C1B2011606 and 2021R1I1A3059984).

Conflicts of Interest: The authors declare no conflict of interest. 


$\begin{array}{ll}\text { Abbreviations } \\ \text { AE } & \text { aerobic exercise } \\ \text { API } & \text { application programming interface } \\ \text { AT } & \text { anaerobic threshold } \\ \text { BG } & \text { blood glucose } \\ \text { BLE } & \text { Bluetooth low energy } \\ \text { BMI } & \text { body mass index } \\ \text { CPX } & \text { cardiopulmonary exercise testing } \\ \text { DAQ } & \text { data acquisition } \\ \text { ECG } & \text { electrocardiogram } \\ \text { FBS } & \text { fasting blood sugar } \\ \text { HRR } & \text { heart rate reserve } \\ \text { I/O } & \text { input/output } \\ \text { ID } & \text { identification } \\ \text { MET } & \text { metabolic equivalents } \\ \text { MVPA } & \text { moderate to vigorous physical activity } \\ \text { RFID } & \text { radio frequency identification } \\ \text { RPE } & \text { ratings of perceived exertion } \\ \text { THR } & \text { target heart rate } \\ \text { VO2max } & \text { maximal oxygen consumption }\end{array}$

\section{References}

1. Benroubi, M. Fear, guilt feelings and misconceptions: Barriers to effective insulin treatment in type 2 diabetes. Diabetes Res. Clin. Pract. 2011, 93, S97-S99. [CrossRef]

2. Berkman, B.J.; Gardner, D.S.; Zodikoff, B.D.; Harootyan, L.K. Social work in health care with older adults: Future challenges. Fam. Soc. 2005, 86, 329-337. [CrossRef]

3. Park, J.C.; Kim, S.; Lee, J.H. Self-care IoT platform for diabetic mellitus. Appl. Sci. 2021, 11, 2006. [CrossRef]

4. Park, J.C. Intelligent Healthcare System for Self-Monitoring of Blood Glucose in Patients with Chronic Diabetes. Master's Thesis, Kangwon National University, Chuncheon, Korea, 28 February 2019.

5. Thent, Z.C.; Das, S.; Henry, L.J. Role of exercise in the management of diabetes mellitus: The global scenario. PLoS ONE 2013, 8 , e80436. [CrossRef]

6. Miele, E.M.; Headley, S.A.E. The effects of chronic aerobic exercise on cardiovascular risk factors in persons with diabetes mellitus. Curr. Diab. Rep. 2017, 17, 97. [CrossRef]

7. Biagi, L.; Bertachi, A.; Quiros, C.; Gimenez, M.; Conget, I.; Bondia, J.; Vehi, J. Accuracy of continuous glucose monitoring before, during, and after aerobic and anaerobic exercise in patients with type-1 diabetes mellitus. Biosensors 2018, 8, 22. [CrossRef]

8. Pan, B.; Ge, L.; Xun, Y.Q.; Chen, Y.J.; Gao, C.Y.; Han, X.; Zuo, L.Q.; Shan, H.Q.; Yang, K.H.; Ding, G.W.; et al. Exercise training modalities in patients with type 2 diabetes mellitus: A systematic review and network meta-analysis. Int. J. Behav. Nutr. Phys. Act. 2018, 15, 72. [CrossRef]

9. Cai, H.; Li, G.; Zhang, P.; Xu, D.; Chen, L. Effect of exercise on the quality of life in type 2 diabetes mellitus: A systematic review. Qual. Life Res. 2017, 26, 515-530. [CrossRef]

10. Zhang, L.Y.; Liu, T.; Teng, Y.Q.; Yao, X.Y.; Zhao, T.T.; Lin, L.Y.; Jin, Q.S.; Jin, Y.J. Effect of a 12-week aerobic exercise training on serum fetuin-A and adipocytokine levels in type 2 diabetes. Exp. Clin. Endocrinol. Diabetes 2018, 126, 487-492. [CrossRef]

11. Yi, T.; Oh, H.I.; Jang, I.H.; Koo, D.Y. Exercise performance test using bicycle ergometer in patients with diabetes mellitus. J. Korean Acad. Rehab. Med. 1997, 21, 414-420.

12. Lee, S.H.; Park, D.H. Blood glucose response to various exercise intensities in children and adolescence with type 1 diabetes. Exerc. Sci. 2014, 23, 139-147.

13. An, K.H.; Min, K.W.; Han, K.A. The effects of aerobic training versus resistance training in non-obese type 2 diabetics. Diabetes Metab. J. 2005, 29, 486-494.

14. Pan, X.R.; Li, G.W.; Hu, Y.H.; Wang, J.X.; Yang, W.Y.; An, Z.X.; Hu, Z.X.; Lin, J.; Xiao, J.Z.; Cao, H.B.; et al. Effect of diet and exercise in preventing NIDDM in people with impaired glucose tolerance: The Da Qing IGT and Diabetes study. Diabetes Care 1997, 20, 537-544. [CrossRef] [PubMed]

15. Oshida, Y.; Ishiguro, T. Exercise therapy for the aged diabetics. Nippon Rinsho Jpn. J. Clin. Med. 2006, 64, 81-86.

16. Adolfsson, P.; Nilsson, S.; Albertsson-Wikland, K.; Lindblad, B. Hormonal response during physical exercise of different intensities in adolescents with type 1 diabetes and healthy controls. Pediatr. Diabetes 2012, 13, 587-596. [CrossRef] [PubMed]

17. Chiang, S.L.; Heitkemper, M.M.; Hung, Y.J.; Tzeng, W.C.; Lee, M.S.; Lin, C.H. Effects of a 12-week moderate-intensity exercise training on blood glucose response in patients with type 2 diabetes. Medicine 2019, 98, e16860. [CrossRef] 
18. Najafabadi, B.A.; Keshavarz, S.; Asgary, S.; Azarbarzin, M. The 8-week aerobic exercise reduces the blood sugar HbA1c and cholesterol levels in women with type 2 diabetes: A single blind randomized controlled clinical trial. Jorjani Biomed. J. 2020, 8, 44-56.

19. Moniotte, S.; Owen, M.; Barrea, T.; Robert, A. Outcomes of algorithm-based modifications of insulinotherapy during exercise in MDI vs. insulin pump-treated children with type 1 diabetes: Results from the tread-diab study. Pediatr. Diabetes 2017, 18, 925-933. [CrossRef] [PubMed]

20. Pawley, D.C.; Damato, A.; Torpiano, J.; Caro, J.X. Physiotherapy for children with type 1 diabetes mellitus (T1DM) in Malta: Effects of exercise and perceptions towards exercise. Physiotherapy 2016, 102, eS19-eS20. [CrossRef]

21. Nguyen, A.L.; Green, J.; Enguidanos, S. The relationship between depressive symptoms, diabetes symptoms, and selfmanagement among an urban, low-income Latino population. J. Diabetes Complicat. 2015, 29, 1003-1008. [CrossRef] [PubMed]

22. Shin, K.O.; Moritani, T.; Woo, J.; Jang, K.S.; Bae, J.Y.; Yoo, J.; Kang, S. Exercise training improves cardiac autonomic nervous system activity in type 1 diabetic children. J. Phys. Ther. Sci. 2014, 26, 111-115. [CrossRef] [PubMed]

23. Haskell, W.L.; Lee, I.M.; Pate, R.R.; Powell, K.E.; Blair, S.N.; Franklin, B.A.; Macera, C.A.; Heath, G.W.; Thompson, P.D.; Bauman, A. Physical activity and public health updated recommendation for adults from the American college of sports medicine and the American Heart Association. Med. Sci. Sports Exerc. 2007, 39, 1423-1434. [CrossRef]

24. Yabe, H.; Kono, K.; Onoyama, A.; Kiyota, A.; Moriyama, Y.; Okada, K.; Kasuga, H. Predicting a target exercise heart rate that reflects the anaerobic threshold in nonbeta-blocked hemodialysis patients: The Karvonen and heart rate reserve formulas. Ther. Apher. Dial. 2021, 1-6. [CrossRef]

25. Nes, B.M.; Janszky, I.; Wisloff, U.; Stoylen, A.; Karlsen, T. Age-predicted maximal heart rate in healthy subjects: The HUNT Fitness Study. Scand. J. Med. Sci. Sports 2012, 23, 697-704. [CrossRef] [PubMed]

26. Fitzgerald, M.D.; Tanaka, H.; Tran, Z.V.; Seals, D.R. Age-related declines in maximal aerobic capacity in regularly exercising vs. sedentary women: A meta-analysis. J. Appl. Physiol. 1997, 83, 160-165. [CrossRef] [PubMed]

27. Balady, G.J.; Arena, R.; Sietsema, K.; Myers, J.; Coke, L.; Fletcher, G.F.; Forman, D.; Franklin, B.; Guazzi, M.; Gulati, M.; et al. Clinician's Guide to cardiopulmonary exercise testing in adults: A scientific statement from the American Heart Association. Circulation 2010, 122, 191-225. [CrossRef] [PubMed]

28. Gellish, R.L.; Goslin, B.R.; Olson, R.E.; McDonald, A.; Russi, G.D.; Moudgil, V.K. Longitudinal modeling of the relationship between age and maximal heart rate. Med. Sci. Sports Exerc. 2007, 39, 822-829. [CrossRef] [PubMed]

29. Sigal, R.J.; Kenny, G.P.; Wasserman, D.H.; Castaneda-Sceppa, C. Physical activity /Exercise and type 2 diabetes. Diabetes Care 2004, 27, 2518-2539. [CrossRef] [PubMed]

30. An, K.H.; Han, K.A.; Min, K.W. Evaluation of physical capacity and proper calculation method of exercise intensity based on measured maximal heart rate in Korean Type 2 diabetics. Diabetes Metab. J. 2005, 29, 479-485.

31. An, K.H.; Han, K.A.; Ku, Y.H.; Ahn, H.J.; Koo, B.K.; Min, K.W. The Effects of Resistance Training on Muscle and Body Fat Mass and Muscle Strength in Type 2 Diabetic Women. Diabetes Metab. J. 2010, 34, 101-110.

32. Shabani, R.; Izaddoust, F. Effects of aerobic training, resistance training, or both on circulating irisin and myostatin in untrained women. Acta Gymnica 2018, 48, 47-55. [CrossRef]

33. Fukuda, T.; Bouchi, R.; Terashima, M.; Sasahara, Y.; Asakawa, M.; Takeuchi, T.; Nakano, Y.; Murakami, M.; Minami, I.; Izumiyama, H.; et al. Ipragliflozin Reduces Epicardial Fat Accumulation in Non-Obese Type 2 Diabetic Patients with Visceral Obesity: A Pilot Study. Diabetes Ther. 2017, 8, 851-861. [CrossRef]

34. Pesta, D.H.; Goncalves, R.L.S.; Madiraju, A.K.; Strasser, B.; Sparks, L.M. Resistance training to improve type 2 diabetes: Working toward a prescription for the future. Nutr. Metab. 2017, 14, 24. [CrossRef]

35. Xiaomi Mi Band. Available online: https://www.mi.com/global/miband (accessed on 24 February 2021).

36. Intel Curie Module. Available online: https://ark.intel.com/content/www/us/en/ark/products/96282/intel-curie-moduleintel-quark-se-soc.html (accessed on 8 July 2021).

37. Digi-Key. Available online: https://components101.com/sensors/hall-effect-sensor-module (accessed on 8 July 2021).

38. Seeed. Available online: https:/ / www.seeedstudio.com/Grove-Ear-clip-Heart-Rate-Sensor.html (accessed on 8 July 2021).

39. Mytinger, M.; Nelson, R.K.; Zuhl, M. Exercise prescription guidelines for cardiovascular disease patients in the absence of a baseline stress test. J. Cardiovasc. Dev. Dis. 2020, 7, 15. [CrossRef]

40. Simms, K.; Myers, C.; Adams, J.; Hartman, J.; Lindsey, C.; Doler, M.; Suhr, J. Exercise tolerance testing in a cardiac rehabilitation setting: An exploratory study of its safety and practicality for exercise prescription and outcome data collection. In Baylor University Medical Center Proceedings; Taylor \& Francis: Abingdon-on-Thames, UK, 2007; Volume 20, pp. 344-347.

41. Harris, G.D.; White, R.D. Exercise stress testing in patients with type2 diabetes: When are asymptomatic patients screened? Clin. Diabetes 2007, 25, 126-130. [CrossRef]

42. Kosinski, C.; Besson, C.; Amati, F. Exercise testing in individuals with diabetes, practical considerations for exercise physiologists. Front. Physiol. 2019, 10, 1257. [CrossRef]

43. Riebe, D.; Franklin, B.A.; Thompson, P.D.; Garber, C.E.; Whitfield, G.P.; Magal, M.; Pescatello, L.S. Updating ACSM's recommendations for exercise preparticipation health screening. Med. Sci. Sports Exerc. 2015, 47, 2473-2479. [CrossRef] [PubMed] 
44. Colberg, S.R.; Sigal, R.J.; Yardley, J.E.; Riddell, M.C.; Dunstan, D.W.; Dempsey, P.C.; Horton, E.S.; Castorino, K.; Tate, D.F. Physical activity/exercise and diabetes: A position statement of the American diabetes association. Diabetes Care 2016, 39, 2065-2079. [CrossRef]

45. Colberg, S.R.; Sigal, R.J.; Fernhall, B.; Regensteiner, J.G.; Blissmer, B.J.; Rubin, R.R.; Chasan-Taber, L.; Albright, A.L.; Braun, B. The American college of sports medicine and the American diabetes association: Joint position statement. Diabetes Care 2010, 33, e147-e167. [CrossRef] [PubMed]

46. Garner, K.K.; Pomeroy, W.; Arnold, J.J. Exercise stress testing: Indications and common questions. Am. Fam. Physician 2017, 96, 293-299. [PubMed]

47. Kim, K.H.; Kim, W.H.; Lee, B.K.; Park, G.W.; Kim, R.; Kim, D.Y. Comparison of rating of perceived exertion and category ratio scale 10 during graded exercise test. J. Korean Phys Edu. 2006, 45, 513-523.

48. Borg, G. Psychophysical bases of perceived exertion. Med. Sci. Sports Exerc. 1982, 14, 377-381. [CrossRef]

49. Borg, G. Borg's Perceived Exertion and Pain Scales; Human Kinetics: Champaign, IL, USA, 1998.

50. Busch, A.J.; Overend, T.J.; Schachter, C.L. Fibromyalgia treatment: The role of exercise and physical activity. Int. J. Clin. Rheumatol. 2009, 4, 343-376. [CrossRef]

51. Kotlin v1.4.32. Available online: http:/ / kotlinlang.org (accessed on 22 April 2021).

52. Jeon, E.; Park, H.A. Development of the IMB model and an evidence-based diabetes self-management mobile application. Healthc Inform Res. 2018, 24, 125-138. [CrossRef] [PubMed]

53. Kim, E.K.; Kwak, S.H.; Baek, S.; Lee, S.L.; Jang, H.C.; Park, K.S.; Cho, Y.M. Feasibility of a Patient-Centered, Smartphone-Based, Diabetes Care System: A Pilot Study. Diabetes Metab. J. 2016, 40, 192-201. [CrossRef] [PubMed]

54. Årsand, E.; Muzny, M.; Bradway, M.; Muzik, J.; Hartvigsen, G. Performance of the first combined smartwatch and smartphone diabetes diary application study. J. Diabetes Sci. Technol. 2015, 9, 556-563. [CrossRef]

55. Osborn, C.Y.; Ginkel, J.R.; Marrero, D.G.; Rodbard, D.; Huddleston, B.; Dachis, J. One Drop I Mobile on iPhone and Apple Watch: An evaluation of HbA1c improvement associated with tracking self-care. JMIR Mhealth Uhealth 2017, 5, e179. [CrossRef]

56. Diabets:M. Available online: https:/ / www.diabetes-m.com/ (accessed on 8 July 2021).

57. WishCare. Available online: https://play.google.com/store/apps/details?id=com.tastylife.wishcare (accessed on 8 July 2021).

58. iCareD. Available online: https:/ / play.google.com/store/apps/details?id=kr.co.mediex.icared\&hl=ko (accessed on 8 July 2021).

59. MyHealthNote. Available online: https:// play.google.com/store/apps/details?id=com.samsungfire.mhn\&hl=ko (accessed on 8 July 2021).

60. BG Monitor Diabetes. Available online: https://play.google.com/store/apps/details?id=com.wonggordon.bgmonitor\&hl=ko\& gl=US (accessed on 18 July 2021). 